$\begin{array}{ll}\text { Italique } & \text { Italique } \\ \text { Poésie italienne de la Renaissance }\end{array}$

IX $\mid 2006$

Varia

\title{
Tragiche eroine. Virtú femminili fra poesia drammatica e trattati sul comportamento
}

\section{Paola Cosentino}

\section{(2) OpenEdition}

\section{Journals}

\section{Edizione digitale}

URL: http://journals.openedition.org/italique/108

DOI: $10.4000 /$ italique.108

ISSN: 1663-4438

\section{Editore}

Librairie Droz

\section{Edizione cartacea}

Data di pubblicazione: 1 giugno 2006

Paginazione: 65-99

ISBN: 2-600-01088-2

ISSN: 1423-3983

Notizia bibliografica digitale

Paola Cosentino, «Tragiche eroine. Virtú femminili fra poesia drammatica e trattati sul comportamento », Italique [Online], IX | 2006, online dal 31 décembre 2009, consultato il 19 avril 2019. URL : http://journals.openedition.org/italique/108 ; DOI : 10.4000/italique.108 
PaOLa Cosentino

T R A G I C H E E R O I N E.

V I R T Ú F E M M I N I L I

FR A POESIA D R A M A T I A

E TRATTATI SUL COMPORTAMENTO 

Tutti accusan le donne, ed io le scuso Se mille volte al dì cangiano amore; Altri un vizio lo chiama ed altri uso: $E d$ a me par necessità del core.

(Mozart - Da Ponte, Così fan tutte) 



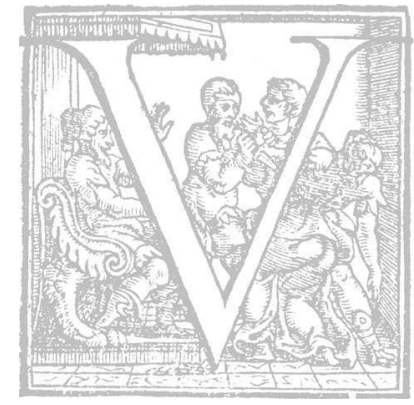

$N$ interesse nuovo, foriero di cambiamenti sia culturali che antropologici, sta alla base della straordinaria attenzione che è rivolta alla donna fra Umanesimo e Rinascimento. ${ }^{\mathrm{I}}$ All'inizio del XVI secolo, la condizione femminile diventa infatti oggetto di una intensa riflessione che tocca i più diversi ambiti e penetra in tutti $i$ generi letterari. Tanto nella novella quanto nella lirica cortigiana, tanto nei trattati quanto nei poemi cavallereschi la donna è al centro di una rinnovata curiosità. Come testimonia il progressivo affermarsi di una vera e propria «querelle des femmes», non soltanto $i$ compiti, ma anche la stessa natura femminile vengono ora interamente rimessi in discussione.

I. Due sono i filoni principali cui è possibile ricondurre la produzione teorica sull'argomento, che del resto affianca la produzione misogina ben presente nella tradizione umanistica: da una parte, $i$ cosiddetti trattati di institutio della donna, generalmente orientati a fornire norme e regole che definiscono una vera e propria grammatica del comportamento, dall' altra, quegli scritti che ne predicano la nobiltà e l'eccellenza, allo scopo di ribaltare il topos tradizionale dell'inferiorità della donna rispetto all'uomo. ${ }^{2}$ Proprio dopo il 1530 saranno dati alle stampe numerosi libelli nati sulla falsariga del celebre trattato di Agrippa, quel De nobilitate et praecellentia foeminei sexus ${ }^{3}$ divenuto capostipite di una generazione di scritti volti a ribadire la dignità della donna. ${ }^{4}$ L'aumento progressivo degli interventi contribuisce, dunque, a una trasformazione dellimmagine muliebre che conosce, durante il secolo, una relativa e stimolante mobilità. Inoltre, la definizione degli spazi e delle funzioni di pertinenza femminile si lega necessariamente alla riflessione sull'istituzione del matrimonio, allinterno della quale la donna acquista importanza come moglie e come madre. La nobilitazione delle nozze è infatti inseparabile dalla riabilitazione dell'immagine della donna, come dimostra il trattato dello spagnolo J. L. Vives, quell'Institutio foeminae cristianae pubblicato nel is23 e anch'esso più volte ripreso proprio nel tentativo di sottolineare l'importanza del ruolo muliebre all'interno della famiglia.' Tuttavia, nonostante la laicizzazione del pensiero favorisca l'espressione delle rivendicazioni femminili, permane un evidente décalage fra la realtà della donna nella società e le rappresentazioni che ne dà la letteratura.

Proprio per mettere in evidenza $i$ mutamenti cui è sottoposta nel secolo la concezione delle virtù e delle attitudini femminili, vorrei qui sondare le connessioni esistenti fra la trattatistica rinascimentale sulla donna e il genere classico 
per antonomasia, risorto grazie alla pubblicażione di Sofocle ed Euripide al principio del secolo e alla vocazione sperimentale di un manipolo di autori gravitanti fra Roma e Firenze: la tragedia. Infatti, proprio l'esperienza ellenizzante di inizio secolo, derivante da quei modelli greci che ebbero una grande propensione per le sentenze morali, rappresenta un terreno privilegiato per la riflessione sul comportamento muliebre. In parte sostituendosi alla parallela produzione teorica in prosa, in parte traendone spunto ora per esaltare il sesso femminile, ora per denunciarne $i$ limiti e le umiliazioni, la poesia tragica cinquecentesca costituisce un interessante luogo di indagine capace di riflettere le più complesse e più spietate dinamiche della società contemporanea, attraverso una raffinatissima maschera letteraria. I drammi cinquecenteschi sono pieni di considerazioni generali affidate tanto ai cori quanto ai personaggi: giudizi che affrontano tutti gli argomenti di maggiore attualità e che non possono ignorare il dibattito sulla posizione della donna. Peraltro, proprio il recupero volgare del dramma antico coincide con la riproposizione di alcuni esempi femminili di grande fascino: figure come Antigone, Alcesti, Medea sono direttamente chiamate in gioco o rivisitate in chiave moderna, riconoscibili nella filigrana delle nuove eroine tragiche che popolano la scena teatrale del Rinascimento. Sottolineando l'importanza "fondatrice" delle prime riscritture drammatiche, si tratterà, quindi, di ripercorrere i testi principali della tragedia cinquecentesca mettendo in evidenza la genesi di un prototipo di eroicità femminile che si definisce già nei primi esperimenti del secolo e che troverà una sintesi suprema nell' ambiguità barocca della Giuditta dellavalliana.

All'origine di questa tradizione modellata sugli esemplari antichi c'è la Sofonisba del Trissino che, come fondamentale archetipo del genere, costituisce il filtro attraverso cui tutta l'esperienza greca viene completamente ripensata. Esso rappresenta il principale incunabolo di quella tragedia fiorentina cui inevitabilmente guarderanno le successive figure femminili, da Orbecche a Canace, da Semiramide ad Adriana, da Marianna fino all'Alvida tassiana. Le eroine eponime dei drammi fiorentini si chiamano Rosmunda, Antigone, Didone, Tullia e considerano loro interlocutrice privilegiata proprio Sofonisba che è un exemplum perfetto di virtù muliebre e che contribuirà a definire il modello della perfetta protagonista tragica. Il personaggio reca in sé $i$ tratti che la tradizione attribuisce alle donne; tuttavia il decoro e la gravità che caratterizzano la regina cartaginese tendono a mettere in evidenza la sua posizione di sovrana: la statura eroica di Sofonisba è data non solo dalla specchiata onestà, ma anche dall'alto livello sociale cui, di fatto, ella appartiene.

Direttamente connesse a quelle predicate dal Castiglione (I528) per la donna di palazzo, le virtù dei personaggi femminili che dalla celebre eroina trissi- 
niana derivano sono le stesse delle matrone romane: pudicizia, continenza, grazia e onestà sono gli attributi necessari a ciascuna delle eroine tragiche qui menzionate. Eppure non tutto coincide. Una più attenta disamina ci porterà, infatti, a verificare l'eccezionale tempra di queste mulieres, la cui forza (morale) è esibita di fronte ai casi avversi con una fermezza e una grinta quasi maschili. Siamo dunque in presenza di un prototipo femminile ascrivibile alla tipologia della "donna illustre" di derivazione boccacciana, già peraltro comparsa ai primordi dell'Umanesimo, come esempio di virtù sia in senso positivo che negativo. Come il Petrarca dei Trionfi fornisce un catalogo delle figure femminili più note della storia e della letteratura antica, ${ }^{6}$ mettendo insieme il mondo greco, quello latino e quello biblico, anche Boccaccio non ha fatto altro che portare sulla scena un congruo numero di donne illustri, raccontandone le memorabili gesta.

Non mi sembra quindi trascurabile il fatto che le tragedie siano più vicine alla prospettiva innovativa dell'autore del Decameron che al trattato del Castiglione, il quale esplicitamente dichiarava di non voler «formar una regina», ma una «donna di palazzo»». Il De mulieribus claris ${ }^{8}$ offre, infatti, un'immagine della virtù e della grandezza femminile molto più "attiva" e pertanto adatta alle scene tragiche. E sarà proprio la concezione boccacciana della claritas femminile - intesa nel suo significato più ampio e applicabile a tutte le donne in possesso di doti straordinarie, a prescindere dall' impiego che esse ne banno fatto - ad affermarsi nella tragedia di primo Cinquecento.

Dotate di una virtu femminile che implica forza e determinazione, queste donne sembrano possedere quelle caratteristiche che in genere si attribuiscono agli uomini. L'eccezionale energia di cui si fanno portatrici è la testimonianza di un coraggio fuori dal comune, che da virtù rischia talvolta di trasformarsi in vizio. Labile è infatti il confine fra bene e male, dal momento che proprio queste tragedie costituiscono il luogo per l'affermazione di una trasgressione di segno femminile. Il dramma diventa infatti il campo di investigazione per la definizione di un modello di donna protagonista dell' azione, la cui condizione ambigua, insieme di "vittima" e di "colpevole", consente l'affermazione di una complessità psicologica nuova. L'eroe o, come in questi casi, l'eroina oscilla perennemente tra il tipo, ovvero un caso illustre, che invita a riflettere sulla precarietà dell'esistenza umana o sull'importanza della virtù, e il modello, inteso come paradigma a cui conformare le proprie azioni. Trissino, ad esempio, potrà menzionare Alcesti, vero e proprio archetipo della sua Sofonisba, come esempio di pudicizia nell'Epistola della vita che dee tenere una donna vedova, ma il rapporto con il personaggio tragico, che secondo Aristotele non deve distinguersi «per virtù e per giustizia» e non deve «cadere per vizio e malvagità, ma per un errore» ( 53 a 7-IO), rimane ancora poco definito. ${ }^{9}$ Non andrà poi trascurato un ulteriore elemento: accanto alle protagoniste dei 
drammi troviamo spesso interlocutrici d'eccezione, che fungono da cassa di risonanza ai lamenti dell'eroina e che, di conseguenza, rendono esplicito il dilemma tragico. Tra $i$ caratteri codificati spicca quello della nutrice, che, proveniente dalla tragedia greca, ma soprattutto dal teatro senecano, è assimilabile alla balia della novellistica e alla mezzana della commedia. ${ }^{{ }^{10}}$ Depositaria di un'etica della moderazione e dell'equilibrio, la nutrice costituisce il controcanto prosaico del dramma, grazie a cui l'unicità e la solitudine dell'eroina risalta ancora meglio. Un altro "personaggio" è il coro, formato dalle fanciulle che, in genere, sono strettamente legate al destino della protagonista: questa soluzione strutturale, derivata da Euripide, nel cui teatro prevalgono $i$ cori di sesso femminile, connota in senso dichiaratamente patetico le tragedie e ne sottolinea le forti implicazioni sentimentali, sullo sfondo di una realtà che riconosce alla presenza muliebre un'importanza mai avuta prima.

2. A Firenze, tra il ISIs e il I530, vengono redatti in volgare alcuni drammi di ispirazione ellenizzante che seguono da vicino l'esperienza del Trissino. Tre sono gli scrittori che operano a stretto contatto e unità di intenti: Giovanni Rucellai (autore della Rosmunda e dell'Oreste), Alessandro Pazzi de' Medici (autore della Dido in Cartagine, ma anche di due importanti traduzioni in volgare, l'Ifigenia in Tauride di Euripide e l'Edipo re di Sofocle) e Ludovico Martelli (autore della Tullia). Nelle loro opere, il recupero sperimentale della tragedia greca, caratterizzata dall' assenza di divisione in atti e da un primo tentativo di applicare le unità pseudoaristoteliche, coincide con la riproposizione di alcuni personaggi femminili di grande fascino, che in tre casi su quattro danno anche il nome al dramma. L'habitus drammatico di queste eroine è mutuato dai personaggi del mito antico: Rosmunda ba il suo modello in Antigone, Ifigenia nell'omonima protagonista della tragedia euripidea, Didone in Medea, Tullia in Elettra. Ciascuna di esse deve però la propria fabula, in parte o completamente, a un'altra tradizione, chi ai repertori di exempla medievali e la storia longobarda (Rosmunda), chi al poema virgiliano (Didone), chi ancora alle storie di Livio (Tullia).

Ogni personaggio femminile risulta essere il frutto di un sapiente lavoro di intarsio, che fonde antico e moderno, leggende derivate dalla storia recente e miti del più lontano passato: se ne ricavano diverse tipologie di donna che tuttavia banno in comune alcuni caratteri fondamentali. L'eccezionale energia di cui sono dotate è la testimonianza di un coraggio fuori dalla norma e, per questo, di per sé sospetto e pericoloso. Se tutta la concezione tragica greca è imperniata dell'ammonimento un $\lambda i \eta_{\eta} v$ «di nessuna cosa troppo» e l'eccellenza minaccia sempre di tramutarsi in tracotanza, nel vizio, le donne sono ancora più suscettibili di oltrepassare i confini. La virtù femminile, ogni qual volta di 
domestica cerca di farsi politica e civile, rischia di trasformarsi in acciecamento, di minacciare la severa razionalità mascbile con un eccesso di passionalità. L'intero sistema politico fiorentino si fonda sulla virtù razionale, e, in fondo, sottilmente calcolatrice, dell'amicizia ${ }^{1 \mathrm{I}}$ con cui le diverse famiglie istituiscono quei vincoli senza $i$ quali non si dà concordia, né «vivere civile». Sulla scena, Rosmunda, Ifigenia, Didone e Tullia sono doppiamente straniere, in quanto donne e in quanto connesse a una realtà "altra", a un universo barbarico e sottilmente perturbante. L'ammirazione per la forza d'animo femminile porta con sé stupore, malessere, paura; anzi il confine fra bene e male rimane, in definitiva, difficile da tracciare con chiarezza. Non a caso, le protagoniste femminili di queste tragedie sono donne che utilizzano la trasgressione per affermare la propria individualità: esse disobbediscono, ingannano, tradiscono, giungono persino ad uccidere. Alla base della raffigurazione convenzionale dell'eroina sta la necessità di introdurre elementi che non sono del tutto conformi alla rappresentazione tradizionale della donna. Tanto è vero che la tragedia si preoccupa di sondare quegli àmbiti che restano fuori dall'indagine compiuta dalla trattatistica contemporanea: cosi, astratti modelli di esemplarità femminile lasciano il posto a figure muliebri esasperate, violente, capaci di portare conflitti insanabili all' interno della comunità e di mettere a repentaglio i fondamenti stessi della vita associata.

Ma, al contrario di quello che avverrà spesso nella tragedia del secondo Cinquecento, qui la donna esce trionfatrice dallo scontro, con l'unica eccezione di Didone a cui è riservato un suicidio trasfigurante. Se infatti si esclude la tragedia di Alessandro de' Pazzi, l'oltranza femminile si rivela ingrediente indispensabile alla ricomposizione della concordia perduta. La donna virtuosa, a contatto con il mondo maschile della politica, si rivela una minaccia benefica, che sovverte l'ordine per favorire una stabilità più duratura. Solo la donna, depositaria di virtù per eccellenza "odissiache" quali l'astuzia e la perseveranza nelle difficoltà, può condurre alla liberazione dal tiranno e dai demagoghi, come se il nemico della città non potesse essere vinto con le tradizionali armi degli nomini. La strada imboccata dai fiorentini produce differenti esempi di figure femminili, ognuno singolarmente impegnato a sostenere le ragioni della sua alterità rispetto al mondo circostante, che visibilmente si contrappone all'estremismo di queste eroine, alla loro sofferta radicalità.

Ma veniamo alle singole tragedie. La prima eroina, Rosmunda, è un esempio di pudore e di delicatezza muliebre che contrasta con il cupo clima medievaleggiante in cui è immersa la vicenda, incentrata sulla sconfitta del popolo dei gepidi ad opera del re longobardo Alboino. Secondo la leggenda riferita da Paolo Diacono, il sourano, durante le nozze con Rosimunda, figlia del re nemico, offre alla donna una coppa di vino ricavata dal teschio del padre. La 
barbara usanza scatena $i$ propositi di vendetta di Rosimunda che, con un astuto stratagemma, riuscirà ad uccidere lo spietato usurpatore.

Rucellai rilegge la storia attraverso l'archetipo classico dell'Antigone di Sofocle. Cosi la fanciulla diviene la custode dei doveri inviolabili della stirpe cui si contrappone la üßpıs tirannica di Alboino-Creonte. Ricalcata sul personaggio di Antigone, Rosmunda dà però vita a un nuovo modello di eroicità femminile, come dimostra il prudente assenso dato alle nozze con il re longobardo, sofferta attestazione di un coraggio che non ba niente in comune con la rigidità della protagonista sofoclea, da cui si differenzia anche per il lieto fine che conclude la sua vicenda. A questo contribuisce in buona parte l'azione dipersuasione svolta dalla nutrice, che insiste sulle pericolose conseguenze di un rifuto alla proposta di matrimonio del sourano da parte della principessa: vengono infatti messe a repentaglio non solo la vita e la libertà delle fanciulle gepide, ma anche la loro castità e il loro onore. Collocato sul fronte opposto della lungimiranza e del buon senso, il personaggio della balia incarna quindi un diverso prototipo femminile, che si fa portatore di valori più solidi quali la saggezza e la prudenza. Gli elementi che contraddistinguono l'interlocutrice privilegiata della giovane figlia di Comundo sembrano derivati da un àmbito contiguo, quello del linguaggio politico machiavelliano: non a caso, il pensiero della donna è costantemente rivolto a quella realtà effettuale che obbliga Rosmunda e le sue compagne ad adeguarsi repentinamente alle "mutazioni" di fortuna. Non solo: $i$ discorsi della donna, evidentemente condizionati dall'intonazione gnomica che appartiene al personaggio, inducono spesso a riflettere sul tema della condizione femminile, cui si addicono le virtù della pudicizia e del decoro. Attraverso le considerazioni della nutrice, viene dunque ribadito il ruolo della donna nella società, che non coincide del tutto con quello incarnato inizialmente da Rosmunda, eroina intrepida e sprezzante del pericolo: le continue allusioni alle violenze e alla brutalità della guerra tendono infatti a sottolineare l'importanza di un atteggiamento pragmatico, soprattutto interessato a tutelare l'incolumità e la rispettabilità della figura femminile. Proprio un significativo momento corale, ${ }^{\mathrm{I} 2}$ consistente in un canto intessuto di lamenti dettati dalla paura e di sentenze sulla dolorosa condizione delle donne sottoposte alle angherie dei vincitori, lascia intendere l'ideologia sottesa al testo: il motivo topico delle fanciulle vittime dei soldati nemici diviene un'occasione privilegiata per esprimere una dura condanna della guerra a vantaggio della pace. Non è un caso infatti che la tragedia si chiuda con un significativo appello alla concordia, palese richiamo al pacificismo universalistico di Leone X.

Tutto il dramma vede contrapposti un universo maschile, fatto di arroganza e di violenza gratuita, e la comunità femminile, formata in genere da donne ridotte in schiavitù. Il lieto fine (che accomuna la Rosmunda all'Oreste $e$ 
alla Tullia) sancisce la vittoria della pietas filiale sul tiranno: il trionfo del bene sul male, della pace sulla guerra, delle donne inermi sulla ferocia dei conquistatori porta impresso un sigillo muliebre che si ricava dalla stessa conclusione della storia, quando Almachilde, promesso sposo di Rosmunda, porterà a compimento l'impresa suggeritagli dalla scaltra nutrice indossando panni femminili.

A un'identica prospettiva si può ricondurre la seconda tragedia del Rucellai, l'Oreste. Ancora una volta, è l'astuzia femminile che consente lo scioglimento positivo della vicenda e la fuga di Oreste e Pilade dalle barbare regioni della Tauride. Ifigenia, ormai recuperata ai sacri valori della famiglia, non senza aver mostrato di possedere una straordinaria virtù "attiva", prenderà il mare con il fratello e l'amico, inseguita dalle stizzite parole di Toante che accomuna il tradimento della fanciulla a quello di alcune celebri figure muliebri dell' antichità. L'accostamento a Medea, ad Arianna, ad Elena varranno ad Ifigenia il riconoscimento del suo ruolo di eroina tragica, che ha convertito la passione d'amore di queste ultime in scaltrezza e ritrovato orgoglio di stirpe. Sourana "decaduta" per convenzione, la figlia di Agamennone troverà la forza di ribellarsi alla sventura che le ha imposto di divenire spietata: grazie ad essa, potrà recuperare la sua antica dignità di donna appartenente a una nobile schiatta di principi.

Con la Didone di Alessandro Pazzi de' Medici, il percorso che qui stiamo delineando si arricchisce di nuove sfumature. In primo luogo, si rafforza la somiglianza con la protagonista della tragedia trissiniana: sacrificate all'arroganza del potere romano, le protagoniste delle due omonime tragedie sono entrambe abbandonate al loro comune destino di vittime. Se, a una prima lettura, la tragica fine della regina cartaginese non sembra affatto paragonabile alla composta morte di Sofonisba che, in procinto di togliersi la vita, trovava serenamente il modo di congedarsi dal mondo, in realtà sono facilmente riconoscibili tutta una serie di motivi che mostrano la straordinaria vicinanza delle due figure tragiche.

La Dido in Cartagine è la prima versione teatrale del IV libro virgiliano, di cui si mettono in scena gli avvenimenti essenziali, riducendo l'azione al momento della partenza dell' eroe troiano. Il dramma si richiama a un conflitto fra le ragioni del privato e quelle del pubblico, fra sentimento e dovere: quando Enea si appresta a far vela da Cartagine, la regina cade in preda alla più totale disperazione perché sta per essere lasciata dall'nomo che l'ba spinta a tradire, colpevolmente, la memoria dell'antico marito. La passione e la rabbia producono un cambiamento psicologico per il quale l'autore si ispira al famoso caso di Medea, come il Pazzi ricorda nella dedica della tragedia indirizzata a Clemente VII. Ancora una volta, il riferimento a un personaggio del mito rafforza il carattere dichiaratamente tragico della protagonista che non solo si 
fa diretta erede della Medea euripidea come di quella senecana, ma risulta costruita attraverso un complesso assemblaggio di elementi eterogenei.

Il Pazzi ha intuito le potenzialità drammaturgiche dell' «amoroso affetto» che domina la regina, colpevole di aver trasgredito gli obblighi imposti dalla vedovanza e dalla responsabilità nei confronti dello Stato di cui è sourana. Per questo, in un crescendo angoscioso che l'avvicina alla Medea della tradizione, Didone non trova pace per l'improvvisa partenza di Enea e lascia che la passione d'amore si trasformi in ossessiva follia, come è più volte sottolineato dalle battute sbigottite ed incredule di tutti coloro che la circondano. Lontani sono gli Dei, consapevoli artefici del destino degli nomini: la regina è sola di fronte all'avanzare di quel furor che la porterà a gettarsi sulla pira, con un gesto estremo che tuttavia la riscatta dalla vergogna subita a causa della seduzione e del conseguente abbandono ${ }^{13}$ da parte dell'eroe troiano. Lavorando su un palinsesto che ben si prestava a una reinterpretazione moderna della vicenda narrata nell'Eneide, lo scrittore fiorentino ba voluto restituire alla regina cartaginese quella dignità eroica che $i$ padri della Chiesa e, sulla loro scorta, il Petrarca dei Trionfi e il Boccaccio del De mulieribus claris avevano ribadito con forza, ${ }^{14}$ insistendo sulla castità e sulla fedeltà alla memoria del marito scomparso: la storia d'amore con il principe esule non viene cancellata, ma anzi fornisce il pretesto per sottolineare l'importanza di un gesto suicida che avvicina Didone agli eroi del passato e che la riscatta agli occhi del suo popolo. Esprimendo una decisa condanna della furia emotiva che ha guidato le sue azioni, la sovrana cartaginese acquisisce una straordinaria coscienza di sé: il Pazzi riesce cosi a creare un personaggio drammatico dal forte spessore psicologico, da cui altri partiranno per dare vita alle figure femminili che animeranno la scena cortigiana del secondo Rinascimento.

A questo medesimo prototipo tragico possiamo ascrivere la Tullia di Lodovico Martelli, dramma in cui la protagonista èpervasa da un furore che non ba più nessuna componente sentimentale, ma che invece deriva da una passione tutta politica. Controfigura di Elettra e come lei mossa da una cupa necessità di vendetta, Tullia è ossessionata dalla riconquista del regno ${ }^{\mathrm{I}}$ che, a suo dire, il padre ha usurpato al suo sposo: al rispetto filiale si sostituisce l'orgoglio di appartenere, attraverso l'unione matrimoniale con il legittimo erede al trono di Roma temporaneamente esiliato, alla dinastia dei Tarquini. Composta sicuramente a Firenze prima del '30, la Tullia sembra essere il prodotto estremo di un'età di trasformazioni e di cambiamenti: proprio quel mito dell'onore e della pudicizia femminile che costituisce il fulcro delle altre tragedie qui prese in esame (si pensi alla pietà di Rosmunda nei confronti del genitore ucciso) entra definitivamente in crisi e viene sostituito da una diversa gamma di valori che lasciano trasparire una nuova visione della politica, senz'altro condizionata dalla coeva riflessione machiavelliana. 
Se Didone, in preda a una sfrenata passione d'amore, decideva di togliersi la vita in nome di una dignità femminile da recuperare e da difendere con ogni mezzo, Tullia si colloca in un altrove "tragico" dove non c'è più posto per $i$ principi del decoro e della regalità. Proprio la trasgressione del codice etico cui si conformavano le altre protagoniste di questi drammi varrà alla Tullia la severa censura di Varchi, che pure apprezzava la qualità poetica di Martelli: «e non posso non maravigliarmi, che uno spirito tanto desto e uno ingegno tanto elevato», dice il letterato fiorentino, «si lasciasse trasportare da non so che a fare una tragedia di persona, sopra la quale non poteva per la scelleratezza sua cadere né compassione, né misericordia, proprio e principal fine della tragedia». ${ }^{16}$ Rispetto ai precedenti esperimenti tragici di Rucellai e di Pazzi de' Medici, questa Tullia, che pure vuole collocarsi sulla scia dell'Elettra greca, risulta opera profondamente ambigua, proprio perché vi prevale la riflessione sul potere e, di conseguenza, sull'innata ferocia di tutti quelli che vi si accostano. Scompare la soglia necessaria a separare il bene dal male: Tullia è personaggio complesso, animato da forze contrapposte, desiderosa di pace e al contempo spinta inesorabilmente verso il compimento di una vendetta che si preannuncia atroce perché del tutto priva di pietà. Recuperando il destino fatale degli Atridi e la vocazione omicida di Medea, Martelli riesce a creare un personaggio inedito, oppresso dalla colpa e tuttavia desideroso di riscatto nei confronti della sventura.

Più volte, le fanciulle che formano il coro ricordano a Tullia la sua condizione di donna «vedova e sola», più volte, la vecchia nutrice fa appello alla necessità di mitigare l'odio, di placare l'ossessione distruttice. Eppure ogni consiglio è vano, perché ciò che contraddistingue la protagonista di questo dramma è anzitutto una personalità forte, volitiva, incapace di rassegnarsi all'attesa di un aiuto esterno, animata come è da una salda coscienza aristocratica e da una non comune passione politica. Non è più l'onore il valore perseguito dall' eroina di questo dramma, ma il soddisfacimento di una pulsione primaria. Per il regno a lungo vagheggiato, Tullia ha ucciso la sorella e il primo marito e ora si scaglia contro i suoi stessi genitori, per riprendersi il ruolo che le compete. Con questa progenitrice di Lady Macbeth, Martelli dà vita a un esempio femminile di empietà e di scelleratezza, che tuttavia non appare meno eroica delle protagoniste di questa prima stagione fiorentina. La virtù femminile, soprattutto in questo caso, sconfina nel vizio e, anzi, la stessa distinzione fondamentale tra "tipo tragico" e "modello tragico" diventa qui del tutto inefficace. Colei che libera dal tiranno è portatrice di metodi non meno efferati: al tiranno esterno si è sostituito un "tiranno" interiore, una passione, una brama di potere che acciecano e disviano le buone qualità di Tullia.

L'aspiraz̧ione alla libertà coincide pure con il desiderio di una tregua, rispetto all'affanno che la domina, mentre il vagheggiamento di una condizione di 
innata purezza si scontra continuamente con la coscienza di vivere in un'età dominata dalla ferocia e dalla violenza. Allinterno di questo universo primordiale in cui tuttavia si riconoscono $i$ tratti di una realtà molto vicina all'autore, Tullia concepisce se stessa soltanto in termini politici, respingendo quelle inclinazioni che possono ricondurla alle mansioni tradizionali della donna. Se Elettra difendeva l'onore del padre, la protagonista della tragedia martelliana recide gli antichi legami familiari per sancire l'importanza di un nuovo vincolo, quello maritale: premio per la fedeltà nei confronti dell'antico consorte sarà il trono, che il padre di lei, Servio Tullio, ha lasciato macchiato di sangue. Sulla finale vittoria di Tullia si staglia, infatti, un'ombra: quella dell'offesa arrecata a una fanciulla (ovvero Lucrezia, moglie di Collatino) da parte di un figlio di Tarquinio il Superbo che sancirà l'avvento della repubblica a Roma. Ai tradizionali modelli femminili che privilegiavano la continenza e la grazia, Martelli oppone questo nuovo exemplum di donna e di sovrana cui non bastano più le tradirionali doti muliebri, superate a vantaggio di un comportamento ferino che lascia trapelare una nuova visione della realtà: ancora una volta, non pare azzardato sottolineare la sotterranea presenza di quel Niccolò Machiavelli con cui il giovane Lodovico aveva in comune amiciz̨ie e frequentazioni. ${ }^{17}$

3. La stagione tragica fiorentina si conclude con la morte della maggior parte degli scrittori che la animarono: fatta eccezione per Luigi Alamanni (spentosi esule in Francia nel I5s6), autore di una pregevole tradurione dell'Antigone sofoclea che aggiunge al panorama appena delineato un altro illustre esempio di eroicità femminile, i protagonisti di questa storia scompaiono tutti prima del I530, senza lasciare eredi. Sarà soltanto una decina d'anni più tardi che il genere tragico rinascerà a nuova vita, trovando una sede adeguata nella Ferrara di Giovan Battista Giraldi Cinzio. ${ }^{18}$

Nel passaggio, la tragedia s'allontana dai greci a vantaggio dei latini, esasperando $i$ toni e raddoppiando gli orrori: il riconosciuto magistero di Seneca (che certo aveva gia fatto scuola all'interno della pur ellenizzante tragedia fiorentina) lascia spazio a un nuovo teatro che tende a riportare in ange $i$ valori della gravitas e del decoro tragico. Insieme alla preferenza accordata al cordovano, il Giraldi, sulle orme del principale modello volgare del genere - la Sofonisba del Trissino - introduce una componente sentimentale che si tradurrà, sulla scena, in un accentuato patetismo derivato anche dalla contaminazione della tradizione senecana con il repertorio novellistico. Proprio in virtù di questa inclinaz̧ione romanzesca, il mondo tragico del Giraldi presenta una galleria di personaggi femminili che hanno perso i tratti eroici delle donne "forti", come Antigone o Elettra, e che sembrano rifarsi a una nuova tipologia muliebre, senz'altro più vicina alle patetiche protagoniste della quarta gior- 
nata decameroniana. Lo dimostra la principale delle tragedie giraldiane, l'Orbecche, che, se da un lato veicola una significativa polemica politica nei confronti del trattato machiavelliano sui principati, dall'altro si presenta come una rielaborazione cinquecentesca della vicenda boccaccesca di Ghismonda. Ma la stessa evoluzione del teatro del ferrarese nella direzione di una tragedia a lieto fine lascia intendere un cambiamento di prospettiva e testimonia la progressiva affermazione di un nuovo gusto che si apre al sentimentale e al cavalleresco, ovvero a quei valori che permeano l'universo del romanzo e che consentono alla corte di rispecchiarsi nelle vicende narrate.

Alla principale tragedia Orbecche rappresentata nel I54I, che narra della truculenta vicenda della figlia di Sulmone, re di Persia, fatta uccidere barbaramente dal padre perché segretamente sposa di un consigliere, fanno seguito la Didone (IS4I) e la Cleopatra (IS43), entrambe messe in scena diversi anni più tardi. Analoghi i destini delle due sourane africane, la cui nobile tempra s'arrende alle leggi d'amore: divenute vulnerabili ed incapaci di reagire alla cattiva sorte, esse sono destinate a soccombere alla dura legge della ragion di stato. Successivamente Giraldi scrive l'Altile (I543) che rilegge la trama della prima tragedia volgendola in positivo, ${ }^{19}$ poiché la principessa che dà il nome alla tragedia e che aveva sposato segretamente un valoroso giovane dai natali oscuri, Norrino, riuscirà ad ottenere la benedizione del fratello, re di Siria. Del I548 sono Antivalomeni (la cui storia si conclude con una grande festa nuziale), mentre nel IssI il Giraldi conclude la Selene che porta in scena ancora una volta una regina, sposa del sovrano di Persia, accusata ingiustamente di adulterio da un cortigiano che tuttavia verrà scoperto e punito. Negli stessi anni, il ferrarese pone mano all'Euphimia (protagonista di un'intrigata vicenda di amori e di ambizioni politiche che si concluderà con il matrimonio della fanciulla con il nobile e saggio re del Peloponneso) e poi all'Arrenopia (dramma ambientato in un contesto "cortese": è la storia di una principessa scozzese, che, dopo il tradimento dell'incostante marito, re di Hibernia, si finge cavaliere, dando luogo a una serie di equivoci, anche "comici", risolti grazie alla tempestiva agnizione finale). Scritta dal Giraldi quando già non era più a Ferrara, l'Epitia è una tragedia dai contenuti decisamente "borghesi" che conducono la protagonista ad accettare il matrimonio con l'assassino del fratello solo per devozione nei confronti dell'imperatore. Decisamente mutato è lo sfondo su cui si muovono le protagoniste di questo teatro: se le loro vicende sembrano costantemente adombrare il dissidio esistente fra il duca di Ferrara e la consorte Renata, in odore di eresia, molti dei motivi portati alla ribalta riflettono le dinamiche negative della corte (l'invidia, il complotto, il tradimento), ma anche $i$ conflitti privati, testimoniati dall'utilizzo di numerosi elementi comici, o "tragicomici", quali il matrimonio o l'adulterio, cui s'aggiungono intrighi, avventure, peripezie che forniranno, poi, 
ampio materiale alla raccolta degli Ecatommiti, dove l'ottica predominante è volta a salvaguardare l'amore coningale e a condannare ogni possibile tentativo di disgregazione e di disordine nella società civile. ${ }^{20}$ Tuttavia le protagoniste di questi drammi hanno perso molta della baldanza e dell'ostinazione delle antiche eroine tragiche: fragilità e passività diventano il loro segno distintivo, come dimostrano numerose sentenze che insistono sulla debolezza muliebre, sentenze tanto più presenti, quanto più significativo è il ruolo del personaggio femminile all'interno della storia. L'immagine della donna protagonista del teatro giraldiano appare dunque più conforme a quella che, negli stessi anni, andavano definendo $i$ numerosi trattati sul comportamento e sull'educazione muliebre, attenti, da un lato, a sottolineare l'importanza assunta dal gentil sesso all'interno della società, dall'altro ad imporre un rigido controllo sociale e a censurare ogni tentativo di autonomia.

Altrettanto frequenti sono poi $i$ luoghi tragici in cui l'eroina lamenta la condizione di infelicità del sesso femminile: così, ad esempio, nell'atto I, scena III della Cleopatra ( $" M a$ la fragilità nostra ci lieva / Anche l'ingegno»), ancora nell'atto I, scena IV dell'Arrenopia ("Questo nostro infelice e miser sesso / Perprivilegio antico o per rea sorte») oppure nell'atto I, scena III della Selene ("Però chi disse, che la donna al mondo / Era uno infelicissimo animale, / Non errò punto, e chi rendeva gratie / A' Dei, perché non l'havea fatto donna, / Non senza gran cagion gliele rendea»). ${ }^{21}$ All'interno di un teatro in cui ruolo della protagonista femminile sulla scena è sempre incarnato da una regina (ovvero dalla moglie o dalla figlia di un re, fatta eccezione per l'Epitia, ultima tragedia giraldiana la cui eroina è una gentildonna), lo spazio dell'autocommiserazione e della coscienza coincide con la momentanea perdita della propria privilegiata posizione sociale: nel pieno rispetto della definizione aristotelica della "colpa tragica", le eroine giraldiane conquistano una relativa autonomia di giudizio proprio nel momento in cui sono costrette a subire l'umiliazione derivata dalla perdita del loro status di sourane e a vivere in un momentaneo regime di indipenden za. Privata costantemente del suo legittimo ruolo, grazie a un meccanismo strutturale ripetuto all'interno di tutte le tragedie con una frequenza che non può non suonare sospetta, la regina giraldiana può effondere così i suoi mesti lamenti e prestare occasionale voce alla disperazione e alla protesta di segno femminile. Tuttavia, il matrimonio (sul quale più avanti ritorneremo) o il ricongingimento finale ristabilirà l'ordine prestabilito e, sancendo il recupero della propria regolare condizione da parte della sourana spodestata, metterà a tacere ogni istanza eversiva cui pure si dava spazio, per siglare invece il trionfo della virtù, della sottomissione, della fedeltà. ${ }^{22}$

A questo stesso repertorio di topoi legati alla situazione femminile nella società si rifanno sistematicamente tutti i testi teatrali modellati sugli archetipi 
antichi (e moderni). Tanto è vero che, in una rapida escursione condotta all'interno delle principali tragedie del secolo, considerate, a torto o a ragione, come gli esemplari più rappresentativi del genere in àmbito cinquecentesco, possiamo facilmente rintracciare più di un richiamo agli aspetti negativi della condizione muliebre. Nell'Orazia di Pietro Aretino (I546), dramma storicopolitico che sposa l'ottica del potere romano, la protagonista femminile, Celia, si lascia andare in preda alla disperazione dopo aver saputo del duello fra Orazi e Curiazi indetto per risolvere la contesa fra Roma e Albalonga. Le sue parole rivolte alla nutrice, che pure derivano da una situazione tutta interna al dramma, evocano tuttavia una realtà universale di sofferenza e di marginalità: "E cosi non sarei la più dolente, / La più infelice isventurata donna / Che persegua tra noi stella maligna / Pianeta iniquo e dispietato influsso» (444-47); e più avanti: «Perché, lassa, non nacqui maschio anch'io?» (476); e ancora: " $" M a$ da che ci son pur femina nata / (Quasi povero fusse l'universo / D'ogni altro esempio di calamitade), / La natura devea, deveva darmi, / In cambio vago de le treccie d'oro, / De le labbra, de i denti, de le ciglia / E d'ebeno e di perle e di rubini, / La sembianza d'un mostro spaventoso» (482-89). ${ }^{23}$ Un altro esempio si ricava invece dall'Adriana del Groto $(1578)$, che intona la consueta lamentatio dell'eroina tragica, qui protagonista di una celebre avventura di amore e di morte che anticipa il Romeo and Juliet shakespeariano: "Che posso dir, se non dolermi al cielo / de lo infelice stato di noi donne, / e invitar tutte in suon flebile unito / a pianger meco le miserie nostre? / Che cessiam dunque, o donne, d'accordarci / a pianger tutte insieme i nostri mali? / Di pigliarci per mano, e disgombrando / il mondo parzïal di noi dolenti, / correre ad affogarci in mezzo a l'acque? / E che vogliamo far qui tra padri duri, / tra crude madri, fra infedeli amanti, / fra sposi alteri, tra tiranni ingiusti, / tra gli nomini, mortali a noi nemici?» (siamo nella terza scena dell' atto III, I5-27). ${ }^{24}$

A fronte di una pattuglia di eroine tragiche, quelle, per intenderci, della prima stagione fiorentina, capaci di superare la loro stessa condizione di vittime in balia di un destino sfavorevole, si collocano figure drammatiche dalle caratteristiche più diverse e tuttavia inclini a riconfermare un codice comportamentale che non potrà essere trasgredito, se non per subire la più efferata delle punizioni. Per questo vengono riproposti, e con maggiore evidenza semantica, $i$ valori ormai codificati dell'onore, della pudicizia e della saggezza, che sono infatti evocati in piu luoghi, specchio di una prassi tragica ormai consolidata, ma anche di un progressivo irrigidimento dei costumi. Nella speroniana Canace, la nutrice si rivolge a Macareo, fratello e incestuoso sposo della protagonista della tragedia, sottolineando l'importanza di uno dei valori fondativi della femminilità: "Onde sol per piacerti / Contra 'l proprio piacere uccider volse / Quella santa onestade / Di cui qual donna è priva / Né 
donna è più né viva» (704-708), ${ }^{25}$ mentre è sempre la balia a ricordare a Celia, nell'Orazia aretiniana che: «Poi che in l'aversitadi si diventa / Prudente e saggia, imparate ora voi, / Dotta d'ingegno, a essere in voi stessa / Saggia e prudente» (atto I, 494-97). Ancora imperniato sulle virtù femminili per eccellenza, quali la castità e la misura, è il dialogo fra la protagonista Marianna e sua sorella Berenice nella tragedia omonima di Lodovico Dolce, in cui pure compare un significativo richiamo all'eroina ebraica più nota, quella Giuditta di Betulia che è esempio illustre di un coraggio tutto maschile fatto proprio da una donna: "Ma io, che debbo far, poi ch'i' son donna? / Quello che fer le giovani animose / Figlie di Belo per gradir al padre / Ch'uccisero i mariti ad uno ad uno? / O seguirò l'esempio de la nostra / Ardita Ebrea, che con la invitta mano / Fece il folle amator del capo scemo?) (atto I, 436-42), suscitando la risposta della sorella Berenice: «Siatevi pur, si come foste sempre, I Casta e modesta, e vi guardate ogn'ora / Di non gli dar un menomo sospetto» (atto I, 463-65).

Ritornano i valori comportamentali legati a un'immagine femminile tradizionale che derivano da una trattatistica progressivamente volta a ripristinare l'ordine all'interno delle istituzioni e a equiparare donne d'alto rango a umili fanciulle avvezze ai lavori manuali, come predica il Dialogo delle istitution delle donne di Ludovico Dolce, modellato sul trattatello del Vives. Insomma: le figure femminili che popolano questa seconda stagione tragica sembrano rinunciare in parte alla volontà trasgressiva dei modelli classici, dal momento che le loro storie si avviano sempre più risolutamente a mettere in scena l'orrore prodotto dalla violenza e dalla ambiguità del potere politico di segno maschile. Alle protagoniste di questi drammi viene restituito il ruolo, a loro più adeguato, di vittime: proprio fra il I545 e il Is63, la Controriforma imporrà, infatti, nuove direttive sociali e, di conseguenza, il ripristino di modelli familiari e sociali secondo $i$ quali una donna può esistere soltanto all'interno dello schema «vergine-moglie-vedova». Se dunque è vero che, nella tragedia, vengono tratteggiate le qualità della sovrana, di quella "donna illustre" balzata in primo piano con l'avvento del classicismo, è altrettanto vero che le caratteristiche progressivamente assunte dalle protagoniste femminili di questo teatro tengono conto del mutato clima ideologico e proiettano sull'antico, favoloso o storico che sia, un presente rigidamente ancorato alla corte e alle sue regole comportamentali. Ciò che avviene all'interno della trattatistica muliebre, volta al progressivo ridimensionamento del ruolo femminile nella società, e del genere tragico, specchio deformante ma fedele ai cambiamenti di costume e di gusto del pubblico al quale si rivolge, ricorda, per analogia, la contemporanea creazione dei primi ghetti ebraici nelle città italiane del Rinascimento: in entrambi $i$ casi, l'istanza normativa - nel primo caso, letteraria, nel secondo, spaziale - corrisponde alla necessità di controllare, 
di incasellare, di reprimere ogni elemento ritenuto portatore di imperfezione o di diversità.

D'altro canto, alla successiva produzione tragica, per la quale possiamo evocare la comoda etichetta di «manierista», secondo la nota definizione di Marco Ariani, ${ }^{26}$ appartengono numerose figure femminili, nobili, principesse e sovrane capaci di sostenere un ruolo attivo, magari condizionato dalla passione o dal desiderio di vendetta: Cleopatra, Altea, Tullia, Semiramide, Acripanda saranno infatti le nuove eroine del dramma tardocinquecentesco che, ispirandosi ai tratti dichiaratamente senecani della prima opera giraldiana (l'Orbecche), ne tradiranno in parte gli assunti morali, portando alla ribalta modelli di donna risolutamente negativi. Tuttavia, questo filone si inserisce in quella generale tendenza all'evasione, fondata sul gusto per l'esotico o sulla riproposizione di valori cavallereschi, che caratterizza la produzione tragica fra il I540 e il I590 e che, invece di dare spazio a una speculazione religiosa sul peccato oppure di riflettere sul conflitto fra individuo e potere, aprirà la strada a una linea patetico-drammatica poi trionfante attraverso l'opera in musica. Paradossalmente più labile e senz'altro più ambiguo si farà il confine fra colpevolezza ed innocenza, fra carnefice e vittima, allinterno di un teatro sempre più legato alle accademie e alle virtuosistiche esibizioni di talento orroroso o romanzesco.

4. Secondo la precettistica aristotelica, il carattere dei locutori della tragedia deve possedere coerenza, verosimiglianza, validità e convenienza. La sfumatura morale che questi attributi assumono all'interno della stessa Poetica ne fanno una sorta di trattato sul comportamento, capace di incanalare, entro una solida griglia, il messaggio ideologico di cui i personaggi si fanno portatori. Fra tutti, la convenienza, ossia il "decoro", intesa come particolare forma di onestà che concerne le manifestazioni esteriori dell'nomo e che, per questo, è avvertita come virtù perfettamente idonea al mondo teatrale, coincide con l'esistenza stessa dei personaggi sulla scena. ${ }^{27}$

Come qualità esterna, in qualche modo "visiva" della moralità, il decoro si colloca fra le virtù richieste alla donna di potere nel Discorso della virtù femminile e donnesca di Torquato Tasso (I587), ${ }^{28}$ primo esempio di una discussione teorica sulle personalità di alto rango appartenenti al sesso femminile che ci riporta, e non è un caso, alle considerazioni fatte in esordio sulla tragedia come luogo di elaborazione di un prontuario comportamentale destinato alla sovrana. Il discorso è infatti una discettazione filosofica - non già un trattato di institutio come si affretta a precisare l'autore - y sulla virtù femminile, relativo, non tanto a una gentildonna privata o a un'industriosa madre di famiglia, quanto a una donna di sangue imperiale che abbia obblighi politici di regno o di governo. Cì̀ su cui Tasso interviene è l'intera virtù della 
donna regia, da cui scaturisce l'assoluta parità di nomo e donna sul piano della virtù cosiddetta "eroica": a questo assunto si collega l'elenco delle donne "elette" della sua epoca, vive o da poco defunte, ma ancora molto presenti nella memoria della collettività. Alla principessa, alla regina (insomma, a chi ha un ruolo di primo piano nella guida di una città e di uno stato) si addicono qualità strategiche, ma soprattutto sono necessarie la prudenza, la fortezza, la leggiadria e, per l'appunto, il decoro.

Il Discorso, che pure ha tutti i limiti dell'encomio cortigiano e della dissertazione posta sotto il segno di Aristotele (per cui risulta piuttosto macchinosa la distinzione fra le due diverse tipologie di donna, l'una condannata a restare chiusa e silenziosa all'interno della dimora domestica, l'altra ammessa ai privilegi di pertinenza maschile, soltanto grazie alle sue doti eccezionali), attribuisce alla donna "illustre" quelle stesse caratteristiche che abbiamo riconosciuto nelle protagoniste delle tragedie fin qui esaminate. L'operetta rivela comunque l'inclinazione tassiana a riconoscere la complessità di una "questione femminile" che sarà oggetto di attenzione anche altrove. Confermandoci l'importanza assunta dalla riflessione sulla natura muliebre nel corso del secolo, ancora una volta nella prospettiva di un rapporto fecondo fra tragedia e trattatistica, Tasso ha infatti dato voce a uno straordinario dibattito sulla condizione della donna proprio nella sua unica tragedia. Nel II atto del Re Torrismondo, lo scambio di idee sul matrimonio fra la regina madre e Rosmonda non solo mostra l'appropriazione, da parte del genere tragico, di temi derivati dal dibattito coevo, ma costituisce un esempio di straordinaria consapevolezza della condizione di inferiorità della donna, all'interno di quell' istituzione che dovrebbe invece tutelare il suo già ridotto spazio esistenziale. Siamo dunque lontani dalle prime tragedie del secolo, dominate da figure muliebri capaci di affermare e soprattutto di far trionfare, attraverso gli exempla tragici di Antigone, di Ifigenia e di Elettra, $i$ valori del femminile: pure, la tragedia tardocinquecentesca continua ad essere il luogo nel quale confluisce la trasgressione, la denuncia, la consapevolezza della subalternità del ruolo muliebre all'interno di una società repressiva e dogmatica.

Tanto è vero che, proprio il discorso pronunciato da Rosmonda ${ }^{29}$ ci permette di affrontare una nuova questione, direttamente legata all'evoluzione dei costumi femminili, ovvero il tema del matrimonio. Una rapida indagine condotta sulle principali tragedie del 'soo consente, infatti, di evidenziare la significativa presenza del motivo nuziale, che si avvia a diventare un topos tragico radicalmente nuovo. Al principio del secolo, nella Sofonisba, viene infatti celebrato il matrimonio fra la protagonista e Massinissa; ${ }^{30}$ a seguire, nella Rosmunda, che proprio sulla tragedia trissiniana è ricalcata, l'eroina è spinta alle nozze con il nemico per necessità strategiche. Quanto alla Didone del Pazzi de' Medici, è evidente che il tema della fedeltà coningale costituisce 
uno dei principali filoni del mito della regina cartaginese; mentre nella Tullia del Martelli viene significativamente sottolineato il valore sacro dell'unione con il marito, per il quale l'eroina tradisce la nobile stirpe cui appartiene. ${ }^{3 \mathrm{I}}$ Ma la centralità concessa al tema del legame nuziale dalla tragedia di ispirazione ellenizzante costituisce un modello operante per tutto il secolo: nell'Orbecche, la figlia del re Sulmone sposa, tacendolo al padre, l'armeno Oronte (ma Giraldi Cinzio farà del matrimonio un tema-cardine di tutte le sue tragedie a sfondo novellistico, che peraltro dimostrano l'origine "narrativa" del motivo), nella Canace, in cui le "nozze" incestuose dei due fratelli gemelli sono la conseguenza della vendetta di Venere, furiosa contro Eolo e Deipoea, nell'Orazia, dove proprio il vincolo matrimoniale che lega Celia a uno dei Curiazi scatenerà la tragedia (cosi la fanciulla al padre: «Ma nel cader del mio sposo sublime, / Io stessa caddi: però che le mogli / Vivano con la vita de i mariti / E moian con la morte de i consorti》, atto II, II83-86); nella Marianna, dramma a tinte fosche fondato sulla gelosia del crudele Erode nei confronti della bella moglie che ne fa un vero e proprio incunabolo dell'Otello shakespeariano, nell'Adriana, in cui il matrimonio segreto fra la protagonista e il principe Latino dà origine alla vicenda ("Ad.: "Le man mi prese, e le sposò l'anella" / Nut.: "Ciò sposarle non fu, ma fu legarle" / Ad.: "Ecco l'anel, che mi lasciò per arra"》), atto I, 205-207), infine nel Re Torrismondo, in cui il tema delle nozze viene affrontato non solo nel colloquio fra Rosmonda e la Regina madre, ma costituisce uno dei motivi fondamentali attorno ai quali è costruita la fabula. Si vuol dire, insomma, che l'insistenza sulla tematica matrimoniale, affatto sconosciuta al dramma antico, autorizza l'ingresso di nuove idee e permette, di conseguenza, una più moderna riflessione sulla società contemporanea, la cui ideologia era già stata peraltro rappresentata dal Tasso nel capolavoro pastorale Aminta. ${ }^{32}$

Accanto a un attento studio della rappresentazione del rito delle nozze, si dourà tener conto del fatto che il tema del matrimonio ha un'origine comica e che esso, per tradizione, ricorre frequentemente nella commedia e nella novella. ${ }^{33}$ E facile, a questo punto, riconoscere la straordinaria commistione di generi di cui la tragedia si fa portatrice, nel momento in cui essa diviene il luogo del confronto, del conflitto, della compresenza di diversi punti di vista. In effetti, proprio partendo dal celebre discorso di Rosmonda sui disagi della vita matrimoniale, possiamo portare alla luce le implicazioni sotterranee di una simile presa di posiz̨ione. Conviene, in primo luogo, rileggere il passo in questione:

ROSMONDA:

«[...] La compagnia de l'uom più lieve alquanto

Può far la noia, e può temprar l'affanno

Onde la vita femminile è grave. 
Ma s'in alcune cose ella n'alleggia, Più ne preme ne l'altre, e quasi atterra, E maggior peso a la consorte aggiunge Che non le toglie in sofferendo. Ed anco Molto stimar si può difficil soma Il voler del marito, anzi l'impero, Qualunque egli pur sia, severo o dolce. Or non è ella assai gravosa cura Quella de' figli? A l'infelice madre Non paion gravi a la più algente bruma Lor notturni viaggi, e i passi sparsi, Ed ogni error ch'i peregrini intrica, La povertà, l'essiglio, e gli altri rischi, E le pallide morti, e i lunghi morbi, Fianchi, stomachi, febri? E s'odo il vero, La gravidanza ancora è grave pondo, E lungo pondo, e doloroso, il parto:

Sì ch'il figliuol, ch'è de le nozze il frutto, È frutto al padre, ed a la madre è peso, Peso anzi il nascer grave, e poi nascendo, Né poi nato è leggiero. E pur di questo, Di cui la vita virginale è scarca, Il matrimonio più n'aggrava e 'ngombra. Che dirò s'egli avien che sian discordi Il marito e la moglie, o se la donna S'incontra in uom superbo, e crudo, e stolto? Infelice servaggio e aspro giogo Puote allor dirsi il suo. Ma sian concordi D'animi, di volere, e di consiglio, E viva l'uno nell'altro; or che ne segue? Forse questa non è penosa vita? Allor quanto ama più, quanto conosce D'essere amata più la nobil donna, Tanto a mille pensieri è più soggetta, Ed a gli affetti suoi gli affetti ascosi Del suo fedel, come sian propri, aggiunge [...] Ma se 'l marito a la gran Madre antica Dopo l'estremo passo al fin ritorna, Ella sente il dolor d'acerba morte, $\mathrm{E}$ seco muore in un medesimo tempo A' piaceri, a le gioie, e vive al lutto.

Onde conchiuderei con certe prove 
Tragiche eroine

Che sia noioso il matrimonio, e grave,

Ch'in lui sterile vita o pur feconda,

L'esser amata od odiosa apporta

Solleciti pensier, fastidi, e pene,

Quasi egualmente».

$$
(\text { I 222-83) } 34
$$

Come giustamente sottolinea M. Guglielminetti, ${ }^{35}$ il passo costituisce un perfetto esempio di come il dialogo tragico possa trasformarsi in un dibattito sulla condizione della donna, degno di figurare in una futura «antologia» sulla questione femminile. Le due interlocutrici, inoltre, si ricavano una zona addirittura prosastica all'interno di una tragedia dove molto spazio è concesso alla riflessione sui grandi temi "privati" e senz'altro romanzeschi dell'amore e dell'amicizia, come del tradimento della fede e dei rapporti fra $i$ sessi. Del resto, Tasso non sottovaluta l'importanza della trattatistica muliebre nella costruzione del suo dramma e potrebbe aver tenuto conto pure di quel Dialogo della dignità della donna che Sperone Speroni aveva scritto, senza tuttavia mai portarlo a compimento, diversi anni prima. ${ }^{36}$ In esso, il letterato padovano, con il quale Torquato aveva avuto un complesso e mai facile rapporto, ${ }^{37}$ affronta l'ingiusta condizione di inferiorità e di servitù cui è costretta la donna all'interno del matrimonio. Il richiamo più diretto è comunque un analogo discorso tenuto da Orbecche nell'omonima tragedia di Giraldi Cinzio:

ORBECCHE :

«Non credo (se lo stato miser guardo Di noi donne) ch'al mondo si ritrovi Sorte sì trista tra l'umane cose Che la nostra infelice non l'avanzi. Noi spesso insin nel ventre de la madre (Pel primo don ch'a noi dà la natura,

Madre a ogn'altro animale, a noi madrigna)

Semo dal padre istesso avute in odio.

Et ove nasce ogn'animale in terra,

Per vil ch'egli si sia, libero e sciolto

(Don che prezzar si dee più che la vita),

Noi, lassa, noi a le catene, a i ceppi,

Oimé, nascemo e a servitù continova.

Perché sì tosto che conoscer nulla

Possiamo, benché tenere fanciulle,

Com'a perpetuo carcere dannate,

Sotto l'arbitrio altrui sempre viviamo 
Paola Cosentino

Con continovo timor, né pur ne lece

Volger un occhio in parte ove non voglia

Chi di noi cura tiene. E dopo quando

Pur devremmo spirar alquanto e avere

Almeno marito a nostra scielta (ancora

Che non mutiam per ciò sorte né stato

Ma sopponiamo il collo a novo giogo),

La madre, il padre od il fratello od altri,

$\mathrm{Al}$ cui severo arbitrio semo date,

Legano il voler nostro e ne conviene

Prender marito a lor volere e ch'essi

Contenti siano. E noi, che con la dote

Comperiamo i mariti e abbiam con loro

Viver fin a la morte, a tal siam date

Che più che 'l dispiacer sempre ne spiace.

E se forse da noi prendiam marito

E se vogliamo far nostro desir contento,

Stiamo a sentenza dura e proviam bene,

Con sommo nostro mal, che cosa importi

Uscir de l'altrui voglie»

$$
(885-921) \cdot{ }^{38}
$$

Riconosciuta fonte di entrambi $i$ passi è il lungo monologo pronunciato da Medea nel primo episodio dell'omonima tragedia di Euripide (230-5I): il poeta greco si scaglia polemicamente contro la società contemporanea, rea di confinare la donna in una posizione subalterna, inferiore a quella dell'nomo sia in senso etico che civile, e di non valorizzarne la profonda umanità per rinnovare un presente ormai privo di valori concreti. ${ }^{39}$ Straniera presso un popolo di cui non conosce gli usi e i costumi, Medea sottolinea la terribile condizione della donna, votata all' infelicità e costretta ad accettare la dura legge della subordinazione agli nomini e del parto doloroso. Il celebre discorso dell'eroina euripidea costituisce dunque il nucleo di partenza dei monologhi delle due protagoniste cinquecentesche, che tuttavia preferiscono concentrarsi su diversi aspetti della questione "femminile" affrontata dalla maga della Colchide. ${ }^{4}$ In effetti, se il Giraldi Cinzio insiste sulla miseria che caratterizza la vita di una donna, sull'odio del padre - che avrebbe preferito un erede maschio, tema peraltro ripreso in alcuni versi dell'Epitia (atto I, scena III: «Se ci nasce una femina, $c i$ duole, / Che nata sia») e della Selene (atto I, scena III: "Oimé, se quando / Nasce a un padre una figlia, egli sen duole / Non è senza cagion la doglia sua. / Che l'infelicità portiam con noi / Dal ventre istesso de le nostre madri») - infine sulla scelta del marito determinata dalle famiglie e dalla ricchezza della dote, Tasso fa invece pronunciare a Rosmonda una severa 
requisitoria contro le false convinzioni relative al matrimonio, che se in parte può «alleggiare» la vita di una donna, ne limita di fatto ogni libertà. Vengono dunque enumerate le noie derivanti dall'unione coniugale: «limpero» del consorte, la "gravosa cura» dei figli, il «grave pondo» della gravidanza e $i$ dolori del parto, la discordia fra moglie e marito, il lutto inconsolabile che segue la morte del compagno. Il testo tassiano sembra dunque fare proprie quelle argomentazioni che venivano spesso utilizzate - anche dagli uomini - per una rappresentazione al tutto negativa del matrimonio: se infatti esso diviene l'espressione più efficace del sistema di alleanze e di rapporti su cui si regge la società rinascimentale, dall'altro, viene costantemente accostato all'ambigua idea del giogo, come può documentare l'immagine, del giogo appunto, contenuta nell'Iconologia di Cesare Ripa. Lo stesso Torquato, autore di una lettera piuttosto nota indirizzata ad Ercole Tasso in cui si sottolineano i piaceri e le gioie del matrimonio, ${ }^{41}$ lascia qui spazio alle ragioni dell'altro, che acquistano un valore ulteriore proprio perché pronunciate da una voce femminile.

Non può sfuggire, del resto, che il prepotente ingresso del motivo coningale nella tragedia cinquecentesca procede in parallelo alla riforma cui viene sottoposto l'istituto delle nozze durante il Concilio di Trento il quale, reagendo alla desacramentalizzazione del matrimonio effettuata nei paesi della Riforma, interviene a regolamentare una materia sfuggente e spesso sottoposta al controllo delle famiglie. Nel febbraio del I563, i lavori dei padri conciliari si preoccupano di rendere il matrimonio una cerimonia pubblica e solenne, celebrata da un sacerdote alla presenza di testimoni: se, in precedenza, le nozze sancivano sostanzialmente un'alleanza fra due nuclei familiari, ora l'unione fra gli sposi segue l'evoluzৃione della società e la presa di posiżione della Chiesa cattolica sottopone a un nuovo regime il vincolo, contribuendo contemporaneamente alla sua secolarizzazione e al suo progressivo affrancamento dalla sfera politica. Insomma, il matrimonio tende a divenire sempre di piu un problema personale di coscienza, proprio nel momento in cui la società si evolve nella direzione di una centralizzazione del potere e dell'affermazione della sovranità statale. Ci sono infatti numerose analogie fra la teoria politica che viene formalizzata alla fine del XVI secolo e la progressiva neutralizzazione delle famiglie nella gestione e nel controllo dei matrimoni. ${ }^{42}$

All'interno della tragedia rinascimentale il tema delle nozze (declinato in vari modi: si pensi al motivo dei connubi infelici o all'unione segreta dei due amanti che scatena la rabbia di Sulmone, nell'Orbecche del Giraldi, o di Semiramide nell'omonima tragedia di Muzio Manfredi) acquisisce un'importanza mai avuta in precedenza ed assume una valenza doppia, riconducibile non solo a una specifica tradizione letteraria (comica e novellistica), ma anche a un costume sociale che va evolvendosi nella direzione di un maggiore controllo della Chiesa cattolica sulla coppia di sposi. ${ }^{43}$ Se ne deduce che proprio la 
tragedia, genere per eccellenza volto a mettere in scena le ambasce di nobili e potenti, testimonia l'importanza via via acquisita dalla riflessione sul matrimonio, che regolamenta, nel suo piccolo, la famiglia e che rispecchia le fun₹ioni assunte dal governo all'interno di uno stato. Tale immagine dell'unione coniugale, derivata dalla concezione umanistica dell'armonia domestica che rispecchia l'armonia della città, affiora dunque tra le righe della produzione tragica del secolo, diventando un motivo fecondo di spunti, legato, da un lato alla rappresentazione del conflitto sociale generalmente provocato dalla ribellione femminile, dall'altro esaltato dalla presenza di vedove devote o infine capaci di recuperare l'onore perduto, come nel caso di Didone, celeberrimo esempio di donna divenuto archetipo tragico per antonomasia, grazie non solo alla storia d'amore raccontata da Virgilio, ma anche alla sua paradigmatica vicenda di vedova che poteva costituire un valido esempio di dirittura morale.

A conclusione di questo rapsodico excursus, volto a mettere in evidenza non solo i cambiamenti cui è stata via via sottoposta la nozione di virtù femminile, ma anche il profondo rapporto esistente fra la tragedia rinascimentale (la cui classica compatezza viene progressivamente messa in crisi dall' approccio sperimentale attuato dai singoli autori nel corso del secolo) e la realtà rappresentata, possiamo tentare alcune rapide considerazioni finali. Come dimostrano gli esempi addotti fin qui, il genere tragico diviene la sede privilegiata per la messa in scena di un mondo animato da affetti e da passioni esasperate che tuttavia veicola un'ideologia "femminile" altrimenti destinata a soccombere. Concedendo spazio a quella riflessione sulla condizione muliebre che permea molte delle opere cinquecentesche e facendo proprie le argomentazioni della trattatistica coeva, la tragedia sembra adombrare un universo magmatico $e$ sfuggente di cui sottolinea la violenza recondita, spesso rivolta verso gli elementi più deboli della società: si pensi, ad esempio, all'ottica decisamente "altra" attraverso la quale viene presentato il matrimonio, spesso oggetto di una satira declinata al maschile che ne mette in evidenza tutti le limitazioni e le difficoltà, sovente determinate dai "donneschi difetti". Assai lontana dalla protagonista, scaltra, ardita o patetica della commedia, la dolente moltitudine di eroine tragiche che popola il nostro teatro rinascimentale dà voce a una lamentatio esistenziale modellata sull'elemento topico antico che tuttavia veicola contenuti nuovi, strettamente legati alla necessità di costruire un sistema drammatico capace di enunciare le verità dimenticate dalla storia e di dare voce, o meglio, forma a un eroismo di segno femminile. Per questo la tragedia risente dei mutamenti cui è sottoposta la società, per questo si fa specchio efficace (come la commedia, del resto, ma da una prospettiva completamente differente) di un privato che diviene pubblico, affidato com'è ai turbamenti e alle ribellioni di moderne sovrane decadute.

Paola Cosentino 


\section{TRAgICHE EROINE}

Le considerazioni qui presentate nascono come appendice a un capitolo del mio Cercando Melpomene. Esperimenti tragici nella Firenze di primo Cinquecento (Manziana, Vecchiarelli, 2003, p. I 38 e segg.), capitolo propriamente dedicato alle protagoniste femminili del teatro tragico fiorentino e, per l'occasione, rielaborato alla luce di nuove indagini e di nuove ricognizioni.

I. Impossibile dare conto in questa sede della sterminata bibliografia esistente sull'argomento. Mi limito a segnalare i contributi cui ho fatto più spesso riferimento nel corso del mio lavoro, ovvero i saggi di C. Fahy, Three early Renaissance Treatises on Women, in «Italian Studies», XI (1956), pp. 30-55, di A. Chemello, Donna di palazzo, moglie e cortigiana: ruoli e funzioni sociali della donna in alcuni trattati del cinquecento, in La corte e il "Cortegiano". Un modello europeo, a cura di A. Prosperi, Roma, Bulzoni, I980, pp. I I 3-32, e il più recente intervento di F. Sberlati, Dalla donna di palazzo alla donna di famiglia. Pedagogia e cultura femminile tra Rinascimento e Controriforma, in «I Tatti Studies», 7 (I 997), pp. I I 9-74. Ma utili si sono rivelate anche le raccolte miscellanee dedicate alla "questione femminile": cfr. Images de la femme dans la littérature italienne de la Renaissance. Préjugés misogynes et aspirations nouvelles, Paris, Université de la Sorbonne Nouvelle, I 980 e Trasgressione tragica e norma domestica. Esemplari di tipologie femminili dalla letteratura europea, a cura di Vanna Gentili, Roma, Edizioni di Storia e Letteratura, I 983 .

2. Una delle prime opere volgari di questo genere è il famoso trattato di Galeazzo Capra intitolato Della eccellenza e dignità delle donne (pubblicato a cura di M. L. Doglio, Roma, Bulzoni, $200 \mathrm{I}, 2^{\circ}$ ed.). Dato alle stampe nel I 525 a Roma e subito dimenticato grazie alla perentoria affermazione del Cortegiano di Baldassar Castiglione, esso sembra nascere all'interno di un sistema dove già molti erano i segnali di interesse nei confronti del mondo femminile.

3. Per il testo latino dell'opera, cfr. Henri Corneille Agrippa, De nobilitate et praecellentia foeminei sexus, édition critique d'après le texte d'Anvers I 529 , préface de Roland Antonioli, Genève, Droz, I990. Tradotto in volgare da Francesco Coccio e pubblicato nel i 544 a Venezia per i tipi di Giolito, il trattato dell'umanista tedesco sottolinea la superiorità femminile in ogni campo, come testimoniano molti esempi tratti dalla storia sacra e da quella profana (Plutarco, Valerio Massimo, Boccaccio). Campionesse di una virtù celebrata dai greci come dai romani sono Artemisia, Alcesti, Cassandra, Ifigenia, Camilla, Cornelia, Lucrezia, Porcia, cui andranno aggiunte eroine bibliche quali Judith, Ruth, Ester.

4. Sulle ambivalenze e sulle contraddizioni interne al discorso rinascimentale sulla superiorità femminile si vedano comunque le considerazioni di F. Danaens, Doxa e paradoxa: uso e strategia della retorica nel discorso sulla superiorità della donna, in «donnawomanfemme», 1985 , nn. 25-26, pp. 27-38 (che riprendono il celebre saggio contenuto in Trasgressione tragica e norma domestica. Esemplari di tipologie femminili dalla letteratura europea, a cura di Vanna Gentili, Roma, Edizioni di Storia e Letteratura, I983, pp. I I-50).

5. Un'interessante disamina dei trattati sul matrimonio è quella offerta da Daniela Frigo nel suo Dal caos all'ordine: sulla questione del «prender moglie» nella trattatistica del sedicesimo secolo (contenuto in Nel cerchio della luna. Figure di donna in alcuni testi del XV T secolo, a cura di Marina Zancan, Venezia, Marsilio Editori, I983, pp. 57-93). Ne emerge un fondamentale spaccato della società tardocinquecentesca preoccupata di garantire l'ordine sociale attraverso la definizione di regole sull'organizzazione della famiglia (aristocratica), destinata a divenire un effettivo centro di potere politico. Il passaggio che si effettua intorno agli anni Cinquanta del secolo e che sancisce la trasformazione di una letteratura volta ad esaltare la nobiltà della donna in una produzione trattatistica a sfondo domestico e familiare appare analogo al ridimensionamento della figura femminile all'interno della tragedia cinquecentesca. Come si vedrà, nonostante la straordinaria presenza di eroine dotate di una tempra virile nei drammi di fine secolo - e penso a Semiramide, a Cleopatra, a Tullia - il destino di queste campionesse di coraggio e di audacia eccezionali non potrà che essere la sconfitta, a tutto 


\section{Paola Cosentino}

vantaggio di un'etica muliebre che privilegia la sottomissione, la ratio, il rispetto di una gerarchia imposta da un potere ancora tutto maschile.

6. Accanto al genere specifico delle biografie femminili, molte sono le opere (fra cui gli stessi Trionfi) in cui sono forniti lunghi elenchi di donne illustri, come gli exempla di tradizione medievale, oppure le storie, le dedicatorie, finanche intere sezioni di celebri capolavori quali il Cortegiano del Castiglione o l'Orlando Furioso di Ariosto. Ne offre un sintetico schema B. Collina nel suo L'esemplarità delle donne illustri fra Umanesimo e Controriforma, in L'esemplarità delle donne illustri fra Umanesimo e Controriforma, in Donna, disciplina, creanza cristiana dal XV al XVII secolo, a cura di G. Zarri, Roma, Edizioni di Storia e Letteratura, I996, pp. 103-19.

7. «Allor il signor Magnifico, voltatosi alla signora Duchessa, - Signora, - disse, - poiché pur così a voi piace, io dirò quello che m'occorre, ma con grandissimo dubbio di non satisfare; e certo molto minor fatica mi saria formar una signora che meritasse esser regina del mondo, che una perfetta cortegiana, perché di questa non so io da chi pigliarne lo esempio» (III iv. La citazione è tratta dall'edizione di BaLdassar Castiglione, Il libro del Cortegiano, a cura di W. Barberis, Torino, Einaudi, I998, p. 260). E più avanti, Castiglione farà dire al Magnifico, in risposta a una battuta polemica del signor Gasparo Pallavicino: «Non sapete voi che Platone, il quale invero non era molto amico delle donne, dà loro la custodia della città e tutti gli altri offici marziali dà agli omini? Non credete voi che molte se ne trovassero, che saprebbon così ben governar le città e gli eserciti, come si faccian gli omini? Ma io non ho lor dati questi offici, perché formo una donna di palazzo, non una regina» (III x, p. 268). Emerge qui, e con chiarezza, il campo specifico di indagine del Castiglione, che si limita a suggerire le norme del perfetto comportamento sociale all'interno della corte che circonda il principe, oggetto, invece, di quei trattati di Institutio di matrice umanistica, latina e volgare. Alla donna non si addicono, almeno all'interno della piccola società aristocratica di cui discetta Castiglione, ruoli di comando: per questo, fra i numerosi esempi di virtù femminile addotti dall'autore, pochi sono quelli in cui viene sottolineata la capacità strategica o l'abilità a governare. Di questa precisa scelta di campo si fa testimonianza efficace l'esclusione della figura biblica di Giuditta, modello di astuzia, ma anche di valore militare, dagli exempla di donne illustri da citare nel trattato: cfr. l'elenco abbozzato in prima istanza dal Castiglione e riportato da G. La Rocca, Un taccuino autografo per il "Cortegiano", in "Italia medievale e umanistica», 23 (1980), pp. 34I-7I, segnatamente a p. 363. Sul terzo libro del Cortegiano si vedano poi V. Finucci, La donna di corte, in «Annali di Italianistica», 7 (1989), pp. 83-103 e C. Scarpati, Osservazioni sul terzo libro del "Cortegiano", in «Aevum», LXVI (1992), pp. 5 19-37.

8. Nel Medioevo, le biografie femminili erano consacrate all'esaltazione di campioni muliebri di santità o di virtù cristiana. Sulla scia di questa consuetudine, ma soprattutto come risposta "femminile" al De viris illustribus di Petrarca, nasce l'opera che inaugura invece il genere specifico delle biografie di donne famose, rigososamente laiche, del mondo antico e moderno (si va da Eva fino alla regina Giovanna): il De mulieribus claris di Giovanni Boccaccio, che si può considerare come la cellula generatrice degli stessi trattati rinascimentali sulla donna.

9. Proficue, a questo proposito, si rivelano le considerazioni tassiane sulla colpa - o semicolpa - tragica esposte nei tardi Discorsi sul poema eroico, che mostrano l'attenzione riservata dal poeta della Liberata alla tragedia greca peraltro analizzata attraverso l'acutissima lente della poetica aristotelica: «Richiede la tragedia persone né buone né cattive, ma d'una condizione di mezzo: tale è Oreste, Elettra, Giocasta, Eteocle, Edippo, la cui persona fu da Aristotele giudicata attissima alla favola tragica» (cfr. TORQUato TAsso, Discorsi dell'arte poetica e del poema eroico, a cura di L. Poma, Roma-Bari, Laterza, I 964, pp. 102-103). Sul rapporto fra queste osservazioni e le postille tassiane al discorso sulla Canace di Faustino Summo (pubblicato a Padova nel I 590), cfr. E. Russo, L'ordine, la fantasia e l'arte. Ricerche per un quinquennio tassiano (I588-1592), Roma, Bulzoni, 2002, pp. I 55-57. Due "mezzecolpe" tragiche risultano 


\section{TRAgICHE EROINE}

poi drammatizzate nel Re Torrismondo del Tasso, stando almeno all'interpretazione che ne offre R. Gigliucci nel suo Giù verso l'alto. Luoghi e dintorni tassiani, Manziana, Vecchiarelli, 2004, alle pp. I I 5-26.

ı. Si pensi alla figura della donna matura, dispensatrice di consigli, come si presenta, ad esempio, nella Raffaella del Piccolomini, modello di una trattatistica filogina in cui prevale una schietta morale edonistica. Un'esauriente schedatura dei personaggi femminili nella commedia è stata realizzata da A. Staüble in occasione di un seminario organizzato presso l'Università di Losanna: i risultati (presentati all'interno del volume Le sirene eterne. Studi sull'eredità classica e biblica nella letteratura italiana, Ravenna, Longo, I996, p. I 5 s e segg.) pervengono alla definizione di quattro tipologie, in parte ereditate da Plauto e Terenzio e, di conseguenza, dalla commedia umanistica (di cui Staüble si è occupato nel La commedia umanistica del Quattrocento, Firenze, Istituto Nazionale di Studi sul Rinascimento, I968), in parte della novellistica medievale. Si tratta delle madri di famiglia, delle fantesche o ancelle, delle mezzane o cortigiane, infine delle giovani donne, che tuttavia non appaiono quasi mai sulla scena per ragioni di decoro e di convenienza ancora praticate nel teatro rinascimentale (si pensi alla Clizia). Proprio a quest'ultima categoria appartengono, tuttavia, le cosiddette "malmaritate", tema che di fatto costituisce «una delle principali deviazioni dal modello plautino-terenziano» (p. I64): oltre al celeberrimo caso della Lucrezia nella Mandragola, possiamo infatti menzionare il personaggio di Oretta nell'Assiuolo di Cecchi, peraltro modulato proprio sul capolavoro machiavelliano. Di pari passo con il motivo del tradimento per infelicità coniugale, si sviluppa il tema della costanza amorosa, che caratterizza la commedia sentimentale e romanzesca, preludio al teatro spagnolo e inglese del XVI secolo. A partire da questa ricca analisi svolta nell'àmbito della commedia, bisognerebbe approfondire il rapporto fra le eroine tragiche e le protagoniste comiche, spesso artefici di riflessioni pessimistiche sulla condizione femminile (come nel caso di Santilla e Fulvia nella Calandria del Bibbiena, di Rita nel Pedante del Belo, di Togna nella Cortigiana dell'Aretino).

I I. La virtù politica maschile per eccellenza è l'amicizia, non a caso celebrata, in contrapposizione al furore amoroso di Didone, da Alessandro de' Pazzi nella lettera dedicatoria del manoscritto della Dido in Cartagine offerto a Clemente VII: lo scrittore afferma infatti che il «syncero et sancto amore d'amicitia partorisce il più delle volte felici successi», mentre «il disordinato et illecito» produce un «lacrymevole exitio». Sullo sfondo, è agevole sgorgere l'influenza di un celebre dialogo di Cicerone, il Laelius de amicitia, in cui lo scrittore latino esalta i valori della probitas e della virtus all'interno di un programma di rinnovamento etico e politico insieme: il ruolo centrale dell'amicizia fra i cittadini di specchiata moralità diviene uno dei postulati di base dell'umanesimo civile fiorentino, in cui tuttavia è riconoscibile quella necessità di rapporti fra le famiglie più illustri che sarà alla base delle alleanze, ma anche delle lotte interne della repubblica.

I 2. «Giorno infelice, al mio mal sì fecondo, / Poi che la libertate / Mi hai tolto e posto in forza al mio nemico! / Quanto mi aggrava al collo questo pondo, / O figliole allevate / $\mathrm{Al}$ viver casto che vi fu sì amico / Che giova el cor pudico, / L'opere iuste, el tanto amare Dio, / L'officio extremo, pio, / Poi che avere' a servire a questi mostri, / Vedove de' mariti e figliuol vostri?» (307-17), cfr. Giovanni Rucellai, Rosmunda, in Teatro del Cinquecento. La tragedia, vol. I, Milano-Napoli, Ricciardi, I997, p. 206. A questa importante silloge, curata da Renzo Cremante, rinvio anche per la ricca nota bibliografica relativa al genere tragico cinquecentesco, e, più specificatamente, ai drammi antologizzati, ovvero, oltre alla Rosmunda del Rucellai, alla Sofonisba del Trissimo, all'Orbecche di Giraldi Cinzio, alla Canace di Sperone Speroni, all'Orazia di Pietro Aretino e alla Marianna di Ludovico Dolce (si vedano le pp. XV-XXXI del I tomo, le pp. I I I9-26 del II e le introduzioni alle singole tragedie). Di recente pubblicazione sono invece gli Atti del Convegno di Studi (tenutosi a Verona fra il I 4 e il i 5 maggio del 2003) su Il verso tragico dal Cinquecento al Settecento, a cura di G. Lonardi e S. Verdino, Padova, Esedra, 2005. 


\section{Paola Cosentino}

I3. Sul tema, che sarebbe interessante analizzare in una prospettiva al "femminile", per intenderci da Didone, Arianna, Medea fino alla protagonista del recente romanzo di Elena Ferrante I giorni dell'abbandono, Roma, E/O, 2002 e che del resto costituisce «uno dei topoi più comuni della letteratura di tutti i tempi», rimando, fra l'altro, al saggio di M. L. Angrisani, Evoluzione del tema dell'abbandono da Properzio a Foscolo, in Letterature comparate. Problemi e metodo. Studi in onore di Ettore Paratore, Bologna, Patron, I98 I, pp. 537-48 (la citazione è tratta da p. 537).

I4. Della storia della regina cartaginese esistevano due differenti versioni, una risalente a Virgilio, l'altra legata invece a un'antica tradizione in cui era del tutto assente la storia d'amore con Enea. Boccaccio, che sul mito di Didone torna spesso nella sua produzione, tiene conto sia del racconto di Giustino (Epitome da Pompeo Trogo) che della vicenda amorosa narrata dall'Eneide: alla regina cartaginese fa allusione, oltre che nel De mulieribus claris, anche nel proemio dell'Ameto, nel Filocolo, nell'Amorosa visione (XXVIII) e nell'Elegia di Madonna Fiammetta (I). Il certaldese ricompone infine le divergenze fra le due tradizioni, una storica e l'altra poetica, nelle Genealogiae deorum gentilium (II, 6o). Ma su questo si veda il ricchissimo volume di P. Bono - M. V. Tessitore, Il Mito di Didone. Avventure di una regina tra secoli e culture, Milano, Bruno Mondadori, I998, p. 92 e segg.

I s. Tale «appitito del regnare» è sottolineato nel commento che Machiavelli fornisce della vicenda in Discorsi III, I (Non vive sicuro uno principe in uno principato, mentre vivono coloro che ne sono stati spogliati). Liquidando la brama di potere di Tullia con una battuta, il segretario fiorentino si preoccupa, in questa sede, soprattutto di condannare la mancanza di prudenza di Servio Tullio, che, dopo essersi illegittimamente impadronito del potere regale, lasciò in vita i parenti più prossimi del precedente re, Tarquinio Prisco.

i6. Cfr. Benedetto Varchi, Opere, Trieste, Lloyd Austriaco, i 858 , vol. II, p. 732-33.

17. Riecheggiano, infatti, celebri affermazioni machiavelliane $\mathrm{i}$ seguenti versi pronunciati da Tullia: «E l'impresa fu giusta, perché nulla / Si puote oprar per acquistarsi un regno / Che le leggi divine o l'altre varchi» (193-95), cfr. Lodovico Marteldi, Tullia, a cura di F. Spera, Torino, RES, I998, p. 9.

18. Per l'Orbecche rimando al primo tomo del Teatro del Cinquecento. La tragedia cit., pp. 26I-448. Una significativa lettura dell'opera dell'intellettuale ferrarese ha condotto R. Bruscagli, in special modo nei saggi contenuti in Stagioni della civiltà estense, Pisa, Nistri Lischi, 1983 (faccio riferimento a La corte in scena. Genesi politica della tragedia ferrarese, pp. I 27-59, e a G. B. Giraldi: comico, satirico, tragico, pp. 16I-86). Ma, dello stesso autore, si veda pure G. B. Giraldi: drammaturgia ed esperienza teatrale, Ferrara, Sate, 1972.

I9. Nel suo Stagioni della civiltà estense cit., Bruscagli parla dell' Altile come di una «riscrittura o una smentita - dell'Orbecche» (p. I 58 ), mentre Corinne Lucas tende soprattutto a sottolineare, nella somiglianza apparente fra le due opere, le numerose differenze esistenti: la seconda tragedia, infatti, non solo si conclude con un lieto fine, ma contiene molti elementi mutuati dalla commedia e del tutto assenti nel dramma del i 54 I (cfr. De l'borreur au "lieto fine". Le contrôle du discours tragique dans le théâtre de Giraldi Cinżio, Roma, Bonacci, I984, p. I I 8 e segg.).

20. Secondo Marzia Pieri, l'importanza assunta dai personaggi femminili nella novellistica giraldiana deriva dal progressivo affermarsi di una nuova spiritualità post-tridentina che attribuisce alla donna un ruolo più attivo e responsabile all'interno del matrimonio o della monacazione (cfr. La strategia edificante degli «Ecatommiti», in «Esperienze letterarie», III, 2, 1972, pp. 43-74). 


\section{Tragiche EROINE}

21. Cito dall'edizione delle tragedie del ferrarese conservata alla Biblioteca Nazionale di Roma: Le tragedie di Messer Giovan Battista Giraldi Cinthio, in Venetia, appresso Giulio Cesare Calcagnini, I583, con segnatura 35. 4. D. 25.

22. Ho qui sommariamente enunciato le considerazioni cui perviene C. Lucas in un suo bel saggio sulla figura della regina nel teatro giraldiano (Le personnage de la reine dans le théatre de Giraldi Cinzio in La corte di Ferrara e il suo mecenatismo. I44I-I598, Atti del convegno internazionale di Copenaghen, maggio I987, a cura di M. Pade, L. Waage Petersen e D. Quarta, Kobenhavn, Museum Tusculanum, I990, pp. 283-300). Prendendo in esame il personaggio della sovrana, la studiosa evidenzia le peculiari caratteristiche che ne fanno un unicum della storia del teatro tragico cinquecentesco. Non è un caso, infatti, che nessuna donna (o meglio regina) abbia un ruolo negativo e che, proprio in questa prospettiva anche Didone e Cleopatra, altrove stigmatizzate per la loro lussuria o la loro ambizione, vengano riabilitate. Escluse, nonostante il loro alto ruolo sociale, dalla politica, queste figure si fanno depositarie dei valori del privato e sono spesso costrette a subire la tirannia di un potere maschile al quale tuttavia reagiscono, rivendicando l'importanza di un atteggiamento passivo e fermo, dichiaratamente opposto alla natura ambigua e cangiante del sovrano. In questo è agevole riconoscere, da un lato, l'adeguamento a un modello di donna che andava imponendosi progressivamente in quegli anni, dall'altro lo stretto rapporto che ebbero queste tragedie con la storia di Ferrara, o meglio con i contrasti sorti all'interno della coppia ducale: non potevano infatti passare inosservati i richiami del Giraldi alla moralità e al rispetto dell'autorità tradizionale in un contesto dove Renata di Francia si era posta visibilmente in contrasto con le posizioni del consorte Ercole II, giungendo a creare una corte alternativa rispetto a quella del duca (ma su questo si veda A. Prosperi, L'eresia in città e a corte, contenuto nel medesimo La corte di Ferrara, cit., pp. 267-8I).

23. Cito dal primo atto dell'Orazia contenuta in Teatro del Cinquecento. La tragedia cit., tomo II, pp. 6i4-i6.

24. Cfr. Il teatro italiano, II, La tragedia del Cinquecento, Tomo primo, a cura di M. Ariani, Torino, Einaudi, 1977, p. 362.

25. Rimando, ancora una volta, alla silloge delle tragedie contenute in Teatro del Cinquecento cit.: si vedano, rispettivamente, per la Canace, pp. 502-503; per l'Orazia, p. 6I5; per la Marianna, pp. 775-76.

26. Cfr. M. Ariani, Tra classicismo e manierismo. Il teatro tragico del Cinquecento, Firenze, Olschki, 1974.

27. Nel suo Dioniso e Tiziano. La rappresentazione dei "simili" nel Cinquecento tra decorum $e$ sistema dei generi, Roma, Bulzoni, 200I, G. Alfano analizza la categoria del decorum quale principio retorico atto a regolare la corrispondenza fra àmbito tematico e registro stilistico all'interno della produzione teorico-letteraria del XVI secolo.

28. Sul discorso tassiano cfr. Dennis J. Dutschke, Il discorso tassiano "de la virtù feminile e donnesca", in «Bergomum», n. 3-4, 1984, pp. 5-28. In realtà, già Antonio Cornazzano aveva dedicato il trattato intitolato Del modo di regere e di regnare (composto fra il I 478 e il I 479 ) ad Eleonora d'Aragona, moglie di Ercole d'Este. L'opera (per la quale cfr. A. Musso, Del modo di regere e di regnare di Antonio Cornazzano: una Istitutio Principis al femminile, in «Schifanoia», 19, 1999, pp. 67-79) fornisce il ritratto della buona regnante, seguendo lo schema tradizionale degli specula principis. Sulla "questione femminile" erano comunque intervenuti Sabatino degli Arienti, con la Gynevera de le clare donne, Filippo Foresti con il De claris mulieribus, Bartolomeo Cogio, con il De laudibus mulierum e lo stesso Cornazzano con il De mulieribus admirandis. 


\section{Paola Cosentino}

29. Giovanni da Pozzo riconduce le parole della fanciulla, che denuncia le difficoltà della vita femminile e, di conseguenza, manifesta il desiderio di ritirarsi in un chiostro, al clima di generale incertezza e ambiguità dominanti nella tragedia tassiana, dove ogni personaggio è fin dall'inizio avviato alla progressiva cancellazione della propria identità, fino al suicidio finale dei due protagonisti (cfr. Dall'Aminta al Torrismondo: manierismo costruttivo e coerenza della tragedia, in Dal Rinaldo alla Gerusalemme: il testo, la favola, Atti del Convegno Internazionale di Studi "Torquato Tasso quattro secoli dopo", Sorrento 17-19 novembre 1994, a cura di D. Della Terza, Sorrento, Eurograf, I997, pp. 49-9I).

30. Si veda, infatti, il racconto delle nozze segrete fra Sofonisba e Massinissa raccontate da un messo a Lelio: «Ma racchetato il vulgo, un sacerdote / Si fece avanti e disse este parole: / "O sommo Giove e tu del ciel Regina, / Siate contenti di donar favore / A queste belle et onorate noze; / E concedete ad ambi lor ch'insieme / Possan godersi in glorioso stato / Fin a l'ultimo dì de la sua vita, / Lasciando al mondo generosa prole." / Dapoi rivolto a la Regina disse: / "Sofonisba Regina, evvi in piacere / Di prender Massinissa per marito, / Massinissa ch'è qui, Re d'e Massuli?’" / Et ella già tutta vermilja in faccia / Disse con bassa voce esser contenta. / Poi questi dimandò se Massinissa / Era contento prender Sofonisba / Per legittima sposa. Et e' rispose / Ch'era contento, con allegra fronte. / E fattosi a la donna più vicino, / Le pose in dito un prezioso anello» (823-43). Nell'annotazione, Cremante riporta le critiche Tasso a un rito che era, in tutta evidenza, anacronistico. Dello stesso genere le nozze descritte da una serva nella Rosmunda, dove peraltro spiccano le analogie con il brano trissiniano appena citato: «E poco stando poi si fece avanti / Falisco e facto ognun tirar da parte, / Cominciò prima a dir certe parole / Laudando il matrimonio; e dicto questo / Si volse a la Regina e le richiese / Si era contenta prender per marito / Lo invictissimo Re de' Longobardi. / Ella con li occhi vergognosi e tardi, / Vermiglia in faccia, riguardando in terra, / Dopo certo silenzio gli rispose / Con tremebonda voce esser contenta. / Quindi, rivolto al Re, simil dimanda / Fece, chiedendo si volea Rosmunda, / Et ei rispose sì senza tardare; / E trattosi di mano un ricco anello, / Lo pose in dito a la Regina nostra» (955-70). Qui il prefetto Falisco sostituisce il sacerdote della Sofonisba, ma compie evidentemente i medesimi gesti, celebrando una cerimonia cattolica. Si vedano, ad esempio, il «vermilja» riferito a Sofonisba e ripreso da Rucellai per Rosmunda (per l'appunto, «vermiglia in faccia»), oppure la «bassa voce» della regina cartaginese che diviene la «tremebonda voce» della principessa gepida; oppure ancora il «prezioso», il «ricco» anello che sigla in entrambi i casi la vicenda. Nella valutazione del rito matrimoniale, bisogna tuttavia tener presente che, almeno fino al Concilio di Trento, le unioni erano sancite da semplici atti notarili, all'occorrenza benedetti da un celebrante. Si trattava infatti di una cerimonia privata che solo più avanti sarebbe stata sottoposta al rigido controllo della Chiesa.

3I. Queste le parole della principessa romana: «Folle come credea / La mia madre ch'io fusse / $\mathrm{Al}$ mio marito avversa, / S'ella uccise pel suo la madre e 'l padre? / Il mio fero parente / Non sapeva che Dio / Assai più d'altro stringe / Il maritale amor con santi nodis (322-29), cfr. Martelli, Tullia, p. i 3.

32. Sulla valenza ideologica delle nozze che concludono la pastorale e che ripristinano l'ordine, sottomettendo Eros alle leggi di Onore, si sofferma infatti S. Zatti nel suo Natura epotere nell" «Aminta», in Studi di letteratura e filologia offerti a Franco Croce, Roma, Bulzoni, I997, pp. I 3 I- 47 .

33. Sul tema delle nozze "letterarie" è da poco uscito il bel volume di Fabio Danelon, $N e ́$ domani né mai. Rappresentażoni del matrimonio nella letteratura italiana, Venezia, Marsilio, 2004. Nel primo capitolo, l'autore si occupa del matrimonio all'interno del genere comico, segnatamente nella Mandragola e nella Clizia del Machiavelli. In merito, si vedano anche alcuni degli interventi contenuti nel volume miscellaneo Le mariage au temps de la Renaissance, éd. par M. T. Jones-Davies, Paris, Klicksieck, 1993. 


\section{TRAgICHE EROINE}

34. Cfr. Torquato Tasso, Il Re Torrismondo, Parma, Fondazione Pietro Bembo - Ugo Guanda Editore, I993, pp. IOI-IO3, a cui rimando anche per la ricca bibliografia sulla tragedia tassiana. Una panoramica più aggiornata è contenuta in R. Gigliucci, Elementi per l'esegesi del Re Torrismondo, in Giù verso l'alto. Luoghi e dintorni tassiani cit.

35. Vedi la sua introduzione a Torquato Tasso, Teatro, Milano, Garzanti, i983, p. XXXVI.

36. Dello Speroni, oltre alla documentata introduzione di M. Pozzi ai dialoghi contenuti nel II tomo dei Trattatisti del Cinquecento, Roma-Napoli, Ricciardi, I 996 (ma, dello stesso autore, si vedano le pagine dedicate allo scrittore padovano in Lingua, cultura e società. Saggi sulla letteratura italiana del Cinquecento, Alessandria, Edizioni dell'Orso, I989, pp. 205-56), si legga anche il Dialogo della cura famigliare, dove appunto l'autore riprenderà, più seriamente, il tema della moglie per sua natura sottomessa al marito. Ma sulle novità contenute nel dialogo rimando alle osservazioni di M. D’Amelia, Marito e moglie. Il dialogo della cura familiare $d i$ Sperone Speroni, in «Memoria», I (I98I), pp. 76-86. Un altro significativo riferimento può essere la Circe di G. B. Gelli, che per l'appunto affida ad una cerva il compito di condannare gli uomini per il riprovevole comportamento tenuto nei secoli nei confronti del sesso femminile. Un'utile disamina di questo dialogo porta la firma di C. Cassiani, $I l$ "discorso" della cerva nella Circe di Gelli, in Roma Donne Libri tra Medioevo e Rinascimento. In ricordo di Pino Lombardi, Roma, Roma nel Rinascimento, 2004, pp. 409-32.

37. Riassume la lunga e tormentata vicenda dei rapporti fra Tasso e Speroni A. Daniele in Nuovi capitoli tassiani, Padova, Antenore, 1998, pp. 177-96. Sulla questione è tornata pure M. T. Girardi, Tasso, Speroni e la cultura padovana, in Formazione e fortuna del Tasso nella cultura della Serenissima, a cura di L. Borsetto e B. M. da Rif, Venezia, Istituto Veneto di Scienze, Lettere e Arti, I998, pp. 63-77.

38. Ancora Giraldi, nell'Arrenopia, fa dire al coro: «Perché noi / Misere donne, se mutiam ben loco / Et stato, non mutiam per questo sorte, / Che, quando siamo vergini, a la Madre / Siamo soggiette al Padre, et a Fratelli, / Et maritate soggiacciamo sempre/ A' mariti, onde in Servitù siam sempre, / Et non proviamo mai la libertate/ Che preciosa è più di ogni thesoro» (atto III, scena vIII, cfr. Le tragedie di Messer Giovan Battista Giraldi Cinthio cit., p. 80 ).

39. Sui significati veicolati dall'inquietante figura della maga della Colchide si vedano comunque le osservazioni contenute in Euripide, Medea. Ippolito, traduzione di R. Cantarella, introduzione, note e commento di M. Cavalli, a cura di D. Del Corno, Milano, Mondadori, 200I, pp. XXXVII-XLI.

40. Nel trattatello di Arcangela Tarabotti intitolato La semplicità ingannata (Leida, Johannes \& Elzevier, I654) si menzionano i rischi del parto e la noia derivata dal doversi prendere cura dei figli come deterrenti del matrimonio. Ma cfr. il saggio contenuto di S. F. Matthews Grieco, Matrimonio e vita coniugale nell'arte dell'Italia moderna, in Storia del matrimonio, a cura di M. De Giorgio e Ch. Klapisch-Zuber, Roma-Bari, Laterza, I996, pp. 25 I-82. Sulla questione del prender moglie intervennero numerosi umanisti: a fronte dei (pochi) sostenitori della condizione matrimoniale, molti furono invece i detrattori, che vollero soprattutto esaltare i piaceri della vita solitaria, immune dai fastidi arrecati dalla presenza femminile. Su questo versante si colloca un trattatello di Giovanni Della Casa, quell'An uxor sit ducenda su cui recentemente sono tornati C. Vecce e P. Pissavino in occasione del convegno svoltosi a Gargnano del Garda fra il 3 e il 5 ottobre del 1996 (Per Giovanni Della Casa: ricerche e contributi, a cura di G. Barbarisi e C. Berra, Bologna, Cisalpino, I997, rispettivamente alle pp. 457-67 e 469-79). Divenuto motivo topico della letteratura burlesca, il tema fu affrontato anche da Ludovico Ariosto in una celebre satira, la quinta, dedicata al cugino Annibale Malaguzzi (un'imitazione polemica dell'operetta tentò poi Ludovico Paterno: riassume la vicenda R. Cacho Casal, Ariosto, Paterno e la satira sul prender moglie: tra imitazione e contestazione, in «Giornale Storico della Letteratura Italiana», CLXXXI, 2004, pp. 86-Io6). 


\section{Paola Cosentino}

4I. La lettera ad Ercole Tasso, datata i 585 (contenuta nel volume XI delle Opere di Torquato Tasso, Pisa, Capurro, I 823, pp. I 38-52, e intitolata Del maritarsi; sarà ristampata nella raccolta delle Lettere di Torquato Tasso, vol. II, a cura di Cesare Guasti, Firenze, Felice Le Monnier, I 853 , pp. 403-20. Ma l'epistola aveva avuto anche un'edizione cinquecentesca: Dello ammogliarsi; piacevole contesa fra i due moderni Tassi, Hercole cioè, et Torquato, gentillbuomini bergamaschi, pubblicata a Bergamo, presso Comin Ventura, nel I 593, e poi ristampata l'anno successivo, che contiene una dedicatoria ad Antonio Bignami, due componimenti di Giovan Battista Licino, infine, oltre alla risposta tassiana, la «declamatione del signor Hercole Tasso, filosofo» intitolata Contro all' ammogliarsi; infine un'orazione dello stesso Ercole dedicata a Maria Soarda. Ho consultato l'edizione conservata presso la Biblioteca Vaticana e segnata Capponi IV 260), è infarcita di citazioni dai classici greci: infatti, non solo vengono ricordate alcune sentenze euripidee a difesa del sesso femminile («la donna suol difender la donna»; «il lodare le virtù della donna in quel modo, che richiede la sua dignità, è cosa da uomo savio, e dotto", Opere cit., p. I 39), ma viene menzionato l'Oeconomicus di Senofonte (di cui si sottolinea «che anche gl'Iddj medesimi ritrovarono questo giogo del matrimonio, oltre l'altre cose, che furono da loro sapientissimamente istituite», p. I4I), Teofrasto, Platone («Platone medesimo ci conforta a generare i figliuoli, ed a nutrirli», p. I42), Masonio filosofo, Aristofane. L'occasione è quella legata alle nozze di Ercole, che aveva, appunto, redatto un'operetta contro il matrimonio, come accenna lo stesso Tasso nell'esordio della missiva: «Io prima intesi che avevate presa moglie, e poi vidi una vostra scrittura, nella quale biasimate non solamente le donne, ma il maritarsi» (p. I38), e che aveva mosso il poeta alle considerazioni contenute nella lettera. L'esaltazione del legame coniugale è strettamente collegato alle lodi nei confronti delle donne: per questo, il poeta della Gerusalemme menziona, in primo luogo, la leggenda del regno delle Amazzoni (che tuttavia Rosmonda, nel discorso appena citato, definisce «feroci donne», v. I 297), e, passando in rassegna il il catalogo delle virtù muliebri, ovvero la temperanza, la modestia, la mansuetudine, la castità, elogia le donne antiche (poetesse come Saffo, ma anche Alcesti, Artemisia, Lucrezia, Porzia e Ipsicratea, che erano già state celebrate nel Trionfo d'amore di Petrarca, o ancora quelle «squadre intiere [che] hanno lasciato glorioso esempio della virtù femminile», p. I47), le donne moderne (Battista da Saluzzo ed Eleonora d'Aragona presso la corte di Ferrara; Isabella e Leonora Gonzaga ad Urbino), infine tutte quelle figure femminili «delle quali non ci è menzione nelle istorie, le quali si nascosero alla fama istessa, che ha tanti occhi, e tante lingue; e la velarono col velo della vergogna» (p. i47). Nella parte finale dello scritto, il Tasso sottolinea l'importanza del vincolo del matrimonio in quanto fondamentale istituzione sociale. Celebrando dunque l'importanza del «legittimo nodo», il poeta compone un vero e proprio inno in prosa, di cui conviene citare almeno un passo, a mio parere particolarmente significativo: «Tu [sott. il matrimonio] cangiasti le oscure spelonche nelle morbide camere, e i freddi monti negli ornati palazzi! Tu facesti lecito quel, che piaceva; ed onesto, quel che si desiderava. Tu ne ponesti dolce legge agli umani piaceri, e lodevol freno a' trabocchevoli desideri [...]» (p. I 50). In questo brano è senz'altro possibile leggere, in filigrana, l'allusione a un noto passo del capolavoro pastorale Aminta, che tuttavia viene riletto alla luce di una visione controriformistica, quella stessa che aveva informato il IV coro del Pastor fido guariniano.

42. Riassumo qui i risultati del recente lavoro di G. Zarri intitolato Recinti. Donne, clausura e matrimonio nella prima età moderna, Bologna, Il Mulino, 200o: un'ampia indagine, condotta a tutto campo sulla condizione femminile sia laica che religiosa fra XVI e XVII secolo (segnatamente alle pp. 203-50).

43. Di questo argomento si occupa, all'interno del volume miscellaneo sulla Storia del matrimonio (Roma-Bari, Laterza, I996, pp. 2 I 5-50) Daniela Lombardi, il quale sottolinea le importanti innovazioni introdotte dalla Chiesa post-tridentina all'interno dell'istituzione matrimoniale. Le unioni vengono rese pubbliche, anche allo scopo di sottolineare l'importanza del sacramento legato alle nozze e negato dai protestanti. Nell'analisi dei mutamenti intervenuti dopo il Concilio di Trento, la storica sottolinea l'avvenuta sacralizzazione del 


\section{Tragiche EROINE}

matrimonio che corrisponde, tuttavia, ad una modernizzazione nonché ad un'istanza di controllo e di disciplina della popolazione. L'intervento della Chiesa tende ad aumentare il suo potere sulla cerimonia matrimoniale, ma anche a favorire il processo di emancipazione del singolo dalla famiglia (cfr. p. 203 e segg.). 